Florida International University FIU Digital Commons

10-29-1996

\title{
Narrative comprehension: semantic and syntactic summary production
}

Jane F. Devick

Florida International University

DOI: $10.25148 /$ etd.FI14062254

Follow this and additional works at: https://digitalcommons.fiu.edu/etd

Part of the Curriculum and Instruction Commons

\section{Recommended Citation}

Devick, Jane F., "Narrative comprehension: semantic and syntactic summary production" (1996). FIU Electronic Theses and Dissertations. 2784.

https://digitalcommons.fiu.edu/etd/2784 


\title{
FLORIDA INTERNATIONAL UNIVERSITY
}

\author{
Miami, Florida
}

\section{NARRATIVE COMPREHENSION: SEMANTIC AND SYNTACTIC SUMMARY PRODUCTION}

A dissertation submitted in partial satisfaction of the requirements for the degree of DOCTOR OF EDUCATION

IN CURRICULUM AND INSTRUCTION by

\author{
Jane F. Devick
}


To: Dr. I. Ira Goldenberg

College of Education

This dissertation, written by Jane F. Devick, and entitled Narrative Comprehension: Semantic and Syntactic Summary Production, having been approved in respect to style and intellectual content, is referred to you for judgement.

We have read this dissertation and recommend that it be approved.

Lynn Berk

Stephen Fain

Paulette Johnson

Nancy Marshall, Major Professor

Date of Defense: October 29, 1996

The dissertation of Jane F. Devick is approved.

Dean I. Ira Goldenberg

College of Education

Dean Richard L. Campbell

Dean of Graduate Studies

Florida International University, 1996 
- COPYRIGHT 1996 by Jane F. Devick

All rights reserved 
I dedicate this dissertation to my daughter, Samantha who has been a great source of joy and hope for me throughout work on this dissertation.

I dedicate this dissertation in loving memory of my beloved father, niece, and sister who are alive in my thoughts and deeds. 


\section{ACKNOWLEDGMENTS}

I wish to acknowledge the contribution of my chairperson, Dr. Nancy Marshall for her total support and encouragement throughout my work on this dissertation. Special thanks are also due to the other members of my Dissertation Committee Dr. Lynn Berk, Dr. Paulette Johnson, and Dr. Stephen Fain.

A special acknowledgment is made in the memory of Dr. Grover Mathewson whose presence was missed during the final stages of this dissertation. 


\section{ABSTRACT OF THE DISSERTATION \\ NARRATIVE COMPREHENSION: SEMANTIC AND SYNTACTIC SUMMARY \\ PRODUCTION}

by

Jane F. Devick

Florida International University, 1996

Professor Nancy Marshall, Major Professor

The purpose of this research was to examine from a syntactic and narrative structure perspective two narrative summary types: a summary with a length constraint and an unconstrained summary. In addition, this research served to develop a multidimensional theory of narrative comprehension.

College freshmen read two short stories written by written by Sake and were asked to write a constrained summary for one text and an unconstrained summary for the other text. Following this the subjects completed a metacognitive questionnaire. The summaries were analyzed to examine transitivity features and narrative structure features. The metacognitive questionnaires were examined to extract information about plot structure, differences between one and two episode stories, and to gain insight into the strategies used by subjects in producing both summary types.

A Paired t- test conducted on the data found that there was a significant transitivity feature mean difference between a constrained summary and an unconstrained summary indicating that the number of transitivity features produced from each summary type were task dependent.

Chi-square tests conducted on the data found that there were proportional differences in usage between plot features and thematic abstract units in an unconstrained summary and a constrained summary indicating that plot features and thematic abstract units produced from each summary type were task dependent.

Qualitative analyses indicated that setting, goal, and resolution are typical within plot organization, there are summary production differences between one and two episode narratives, and subjects do not seem to be aware of summary production strategies.

The results of this research have implications for comprehension and writing instruction. 
I. INTRODUCTION ....................... 1

Purpose of Research . . . . . . . . . . . . . . 2

The Research Described . . . . . . . . . . . . . . . 4

Definition of Terms . . . . . . . . . . . . . . 6

II. REVIEW OF RELEVANT LITERATURE . . . . . . . . . . . . 7

Relevant Linguistic Literature . . . . . . . . . . . . . 7

Relevance of Transitivity ................. . . 7

High Transitivity . . . . . . . . . . . . . . 12

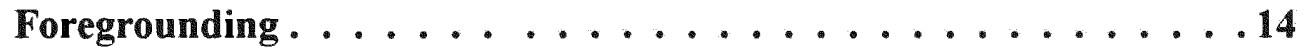

Relevant Cognitive Science Literature . . . . . . . . . . 15

Macrorules for Comprehension and Summary Writing . . . . . . 15

Summary and Comprehension Production Model Operation . . . . 16

The Macrorules . . . . . . . . . . . . . . . . . . 17

The Works of Brown and Day and Marshall. . . . . . . . . 18

Synthesis of Macrorules and Transitivity . . . . . . . . . 20

Relevant Theories of Narrative Literature . . . . . . . . 22

Story Grammars . . . . . . . . . . . . . . . . 22

Relevant Artificial Intelligence Literature . . . . . . . . . . 29

Concrete Schemata . . . . . . . . . . . . . . . 29

Abstract Schemata . . . . . . . . . . . . . . 31

Thematic Abstract Units . . . . . . . . . . . . . . . . 32

Multidimensional Theory of Narrative Comprehension . . . . . 35

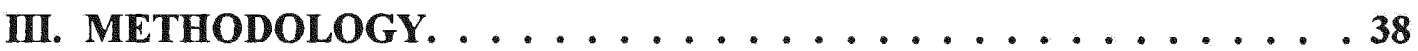

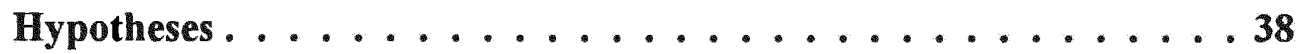

Null Hypotheses . . . . . . . . . . . . . . . . . 39

Research Hypotheses . . . . . . . . . . . . . . . 39

Design . . . . . . . . . . . . . . . . . 43

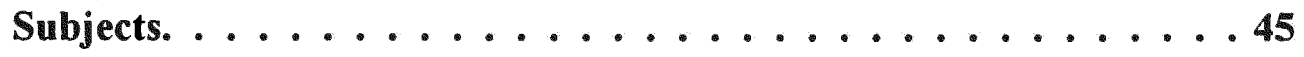

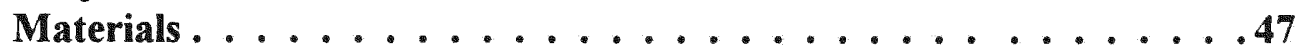

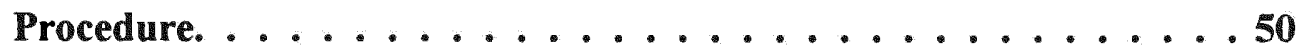

Scoring Procedure. .................... . . . 53

Story Scoring Rules . . . . . . . . . . . . . 53

Transitivity Scoring Rules. . . . . . . . . . . . . 54 
IV. RESULTS. . . . . . . . . . . . . . . . . . . . . . . . . . . . .

Phase I. . . . . . . . . . . . . . . . . . 59

Phase II. . . . . . . . . . . . . . . . . . . 63

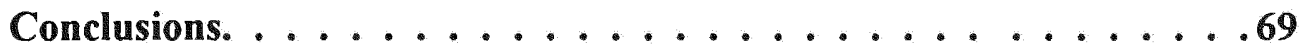

V. QUALITATIVE RESULTS. . . . . . . . . . . . . . 70

Semantic Features of the Unconstrained Summaries. . . . . . . 70

Syntactic Features of the Unconstrained Summaries. . . . . . . . 74

Semantic Features of the Constrained Summaries. . . . . . . . .74

Syntactic Features of the Constrained Summaries. . . . . . . 75

Metacognitive Questionnaire. . . . . . . . . . . . 76

Responses to Question 1. . . . . . . . . . . . . . . . 77

Responses to Question 2. . . . . . . . . . . . . . . 78

Responses to Question 3. . . . . . . . . . . . . . . . 79

Responses to Question 4. . . . . . . . . . . . . . . . . 80

Responses to Question 5. . . . . . . . . . . . . . 81

Summary of the Qualitative Findings. . . . . . . . . . .81

VI. GENERAL DISCUSSION. . . . . . . . . . . . . . . 83

The Questions Answered. . . . . . . . . . . . . . . . 83

Multidimensional Theory of Narrative Comprehension. . . . . . .85

Semantic Story Understanding. . . . . . . . . . . . . 87

Syntactic Features of Story Understanding. . . . . . . . . . 90

Implications. . . . . . . . . . . . . . . . . . . . . . . .

Limitations. . . . . . . . . . . . . . . . . .93

Further Research. . . . . . . . . . . . . . . . . . . . . . . . . . . . .

Summary. . . . . . . . . . . . . . . . . . . 94

A Concluding Statement. . . . . . . . . . . . . . . . .

REFERENCES. . . . . . . . . . . . . . . . 96 APPENDICES

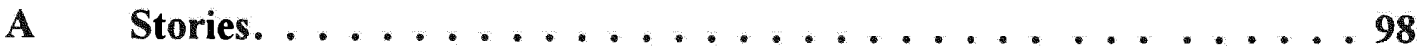


B The King and his Shirt ................. 107

C Sample of Experimental Packet. ............. 108

D Story Scoring Procedure Samples . . . . . . . . . . . . 122

E Transitivity Scoring Procedure Samples . . . . . . . . . 123

F Table 1 Constrained Summary Thematic Abstract Unit by Treatment (1-4) . . . . . . . . . . . . . . . 125

Table 2 Constrained Summary Plot by Treatment (1-4) . . . . . . 126

Table 3 Constrained Summary Theme by Treatment (1-4) . . . . . 127

Table 4 Constrained Summary Other by Treatment (1-4) . . . . 128

Table 5 Unconstrained Summary Thematic Abstract Unit by Treatment (1-4) . . . . . . . . . . . . . . . . . 129

Table 6 Unconstrained Summary Plot Unit by Treatment (1-4) . . . 130

Table 7 Unconstrained Summary Theme by Treatment (1-4) . . . . 131

Table 8 Unconstrained Theme by Story Order . . . . . . . . . . 132

Table 9 Unconstrained Summary Other by Treatment (1-4) . . . . 133

Table 10 Analysis of Variance for Constrained Summary

Transitivity by Treatment $(1-4) \ldots \ldots \ldots 134$

Table 11 Analysis of Variance for Unconstrained Summary

Transitivity by Treatment $(1-4) \ldots \ldots . \ldots 135$

G Transitivity Variability by Constrained Summaries . . . . . . . 136

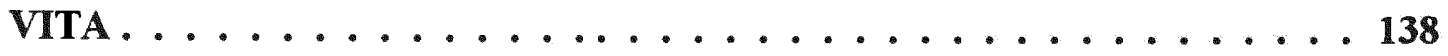




\section{LIST OF TABLES}

TABLE

PAGE

1 Transitivity Features According to Hopper and Thompson . . . . . 8

2 Properties of Individuation . . . . . . . . . . . . 12

3 Design for Independent Variables Used in Data Analysis . . . . . . 44

4 Age English Was Learned and Subject Native Language by Age . . . 47

5 Design for Independent Variables Used in Data Analysis . . . . . . 60

6 Transitivity Features Means by Summary Type and Standard Deviation . . . . . . . . . . . . . . . . 63

7 Percents and Proportion of Usage of Thematic Abstract Units in Summaries. . . . . . . . . . . . . . . 65

8 Percents and Proportion of Usage of Plot in Summaries . . . . . . 66

9 Percents and Proportion of Usage of Theme in Summaries . . . . . 67

10 Percents and Proportion of Usage of Other in Summaries . . . . . 68

11 Percentages of Usage of Thematic Abstract Units and Plot by Summary Type . . . . . . . . . . . . . . 68

12 Number and Type of Semantic Story Feature Contained in the Unconstrained Summaries . . . . . . . . . . . . . .71

13 Number and Type of Semantic Story Feature Contained in the Constrained Summaries . . . . . . . . . . . . 75

14 Subjects Question 1 Category Type and Number of Responses . . . . 77

15 Subjects Question 2 Category Type and Number of Responses . . . . 79

16 Subjects Question 3 Category Type, Number of Responses, and Summary Type . . . . . . . . . . . . . 80 


\section{LIST OF FIGURES}

FIGURE

PAGE

1 Organization of Story Grammar . . . . . . . . . 23

2 Generic Story Structure ................. 24

3 Semantic and Syntax Strategies Used by Readers During the Production of a Summary Task . . . . . . . . . . 86 


\section{Chapter I}

\section{Introduction}

The comprehension of narratives is a complicated process. Over the last twenty years various theories of narrative comprehension have been developed. Most theories of narrative discourse are grounded in only one field of study such as either in the cognitive sciences (Kintsch \& van Dijk, 1977; Stein \& Glenn, 1979; Thorndyke,1977), syntax (Givon, 1984; Hopper \& Thompson, 1980; 1984) or artificial intelligence (Schank \& Abelson, 1977; Seifert, Dyer \& Black, 1986). Theories of narrative representation from each of these fields of narrative study postulate how the development of a concept of story is either stored in the reader's memory or cognitively constructed by the reader. However, there is no theory of narrative representation that begins to tie together the empirical observations made by those in the fields of syntax, cognitive science, and artificial intelligence. According to Graesser, Golding \& Long, (1991) there is a need to develop a multidimensional theory of narrative comprehension to examine the complicated process of narrative comprehension.

Since narrative comprehension is a complicated process, the question arises as to how a reader's narrative comprehension should be examined. The purpose of this research is to examine, from a multidimensional perspective, two narrative summary types: a constrained summary and an unconstrained summary. A constrained summary contains from five to fifteen words that represent narrative understanding, and an unconstrained summary contains at least three sentences that represent narrative understanding.

A predominant method of examining postreading narrative comprehension has been the use of an unconstrained summary (Brown \& Day, 1983). Problems arise from the nature of an unconstrained summary because unconstrained summaries may inhibit students whose ability to write is limited. Thus, there is a need to expand and examine methods of narrative comprehension that avoid these writing ability pitfalls. Writing ability refers to generating a cohesive group of sentences, it does not refer to abstract versus concrete representations of 
text understanding. One such method of examining narrative comprehension is through the use of a constrained summary (Marshall,1984, 1994). A constrained summary of a narrative relies less on the writers composition ability in contrast to an unconstrained summary. It is hypothesized that a constrained summary produces elements of story structure and syntactic features different from an unconstrained summary. This is because the form of a constrained summary forces severe coordination of narrative information while simultaneously preventing very superficial responses. This is in contrast to an unconstrained summary which promotes general narrative information while simultaneously producing superficial responses. Thus, each type of summary provides different ways of representing the meaning of a story and are available as filters to show what has been comprehended.

\section{Purpose of Research}

There are four major purposes of this research. The first purpose of this research is to examine semantic story structure features and syntax features produced by each summary type. This study demonstrates that syntax is important in the study of narrative structure and has been neglected in previous narrative structure research. Second, this research examines the syntactic choices made by the reader when producing a summary. Third, this research examines the semantic choices made by the reader when producing a summary. Fourth, this research will serve to develop a multidimensional narrative comprehension theory. The multidimensional theory for comprehension incorporates works from the areas of syntax, cognitive science, and artificial intelligence. Fifth, this research will begin to examine narrative comprehension processes and narrative comprehension products.

To support the first and second purpose, the works of linguists Givon (1984) and Hopper and Thompson $(1980,1984)$ provide a syntactic and a research foundation that support the hypothesis that an unconstrained summary contains the syntactic and discourse features of high transitivity and foregrounding and a constrained summary contains discourse features that are low in transitivity. In narrative study most discussion never gets down to the 
level of syntax. This study specifically examines the syntactic phenomena of transitivity as it relates to narrative structure. High transitivity refers to clauses that contain dynamic/action verbs, agentive subjects, and totally affected concrete direct objects while low in transitivity refers to clauses that contain mental state, stative, copular verbs, abstract subjects, and unaffected direct objects. The use of transitivity features reflects syntactic strategies used by readers to produce narrative discourse.

To support the third purpose, the works of Kintsch and van Dijk (1977) provide a semantic summary writing model and a research base for discourse summary production differences. It is suggested that a reader applies the construction macrorule strategy when producing a constrained summary and the reader applies the generalization and integration macrorule strategy when producing an unconstrained summary. Also, the works of Brewer and Lichtenstein (1982), Many (1991), Rosenblatt (1986), and Seifert, Dyer, and Black (1986), and Thorndyke (1977) provide a semantic theoretical and research foundation to support the hypotheses that an unconstrained summary contains plot features to foreground the action event parts of narrative comprehension while a constrained summary contains thematic abstract units that represent abstract representations of narrative comprehension. Further, the works of Schank (1982) and Seifert, Dyer, and Black (1986) will be used to support the notion when producing a constrained summary the reader uses certain semantic strategies which produce thematic abstract units while when producing an unconstrained summary the reader uses certain semantic strategies which produces a greater percentage of concrete plot features. To support the fourth and fifth purposes the works from the areas of linguistics ( Givon, 1984; Hopper \& Thompson, 1980, 1984), cognitive science (Kintsch and van Dijk, 1977; Thorndyke, 1977) and artificial intelligence (Seifert, Dyer, \& Black, 1986) will be unified to develop a theory of narration.

In sum, this research will serve to examine story comprehension as well as examine comprehension production pattern differences between constrained summaries and 
unconstrained summaries. The theoretical underpinnings for the research come from the fields of linguistics, cognitive science, and artificial intelligence.

This research is based upon several important assumptions generally accepted by those studying narrative comprehension and syntax.

1. A concept of narration is either stored in memory or cognitively constructed.

2. A concept of narration is organized in personal knowledge structures (schemata) that are activated by readers during the comprehension proves.

3. The structure of narratives is separable from the content of narratives.

4. Both the story features and syntactic feature of narrative summaries are measurable.

5. A constrained summary and an unconstrained summary are considered to be forms of discourse.

6. Summary content and structure provide insights into the processes that were used to create the summary.

\section{The Research Described}

The research to be described here is an investigation of story summary and comprehension production processes and products which are based upon these six assumptions.

College-age freshmen were asked to read two short stories written by Sake (H.H. Munroe) and write a constrained summary for one story and an unconstrained summary for the other story. Following the summaries, they were asked to complete an open-ended questionnaire intended to cause them to think about the differences between both types of summaries. To distribute any effect of story order and summary order, story order and summary order were counterbalanced so that four story order/summary order treatment groups were formed. These were (1) story one constrained summary, story two unconstrained summary; (2) story one unconstrained summary, story two constrained 
summary; (3) story two constrained summary, story one unconstrained summary; (4) story two unconstrained summary, story one constrained summary. Both constrained and unconstrained summaries were examined for of plot, theme, and thematic abstract units and for syntactic features of transitivity. Recalls were examined for story structure elements of plot, theme, and thematic abstract units and syntactic features of transitivity. The instrumentation measures used to examine the summaries were developed by an application of the works of experts in the fields of syntax (Hopper \& Thompson, 1984), in the field of cognitive science (Thorndyke,1977), and in the field of artificial intelligence (Seifert, Dyer, \& Black, 1986).

This research was designed to answer the following questions.

1. How do the story structure features of a constrained summary and an unconstrained summary differ? Is there a consistent pattern in these differences?

2. How does the transitivity of a constrained summary and an unconstrained summary differ? Is there a consistent pattern in the differences?

3. What do the subjects report about their processing of a constrained summary and an unconstrained summary?

4. Are the differences in story features and syntactic features produced by both summaries task dependent?

Answers to these questions should provide insight into the nature of narrative comprehension patterns produced by two summary types and into the syntactic and semantic strategies used by subjects during the production of both summary types.

The details of this research will be presented in the subsequent chapters of this dissertation. Chapter II of this dissertation is devoted to the review of relevant literature in the areas that underlie the study. These include the areas of, syntax (Givon, 1984; Hopper \& Thompson, 1980, 1984), cognitive science (Kintsch \& van Dijk, 1977), narrative theories (Brewer \& Lichtenstein; Many, 1991; Rosenblatt, 1986; Thorndyke, 1977), and artificial 
intelligence (Schank, 1982; Seifert, Black \& Dyer, 1986). Chapter III contains a detailed explanation of the design and the specific hypotheses to be tested for this research. In Chapter IV, the results of the research are presented. In Chapter V, a qualitative analysis of the subjects metacognitive questionnaire and story features produced for each summary type are presented and discussed. Chapter VI contains a general discussion of results and suggestions for further research as well as practical applications of this research. The following is a section of definitions.

Definition of Terms

1. Discourse: Communication of thoughts by words.

2. Narration: A story of events or experiences.

3. Constrained Summary: A one sentence summary that contains between five and fifteen words.

4.Unconstrained Summary: A multiple sentence summary that contains three or more sentences

Narrative comprehension has been an area of active interest for researchers from different fields. However, for the most part empirical observations about narrative comprehension processes and products have been in the areas of cognitive science and artificial intelligence. There is a need to examine narrative comprehension that begins to include other fields of study. As a result, relevant literature from the fields of linguistics, cognitive science, and artificial intelligence shall be reviewed here. 


\section{Chapter II}

\section{Review of Relevant Literature}

\section{Relevant Linguistic Literature}

The purpose of the linguistic review of relevant literature in the area of syntax is to develop a foundation for an analysis of the two summary types, constrained and unconstrained, so that any structural differences in the two could be discovered. A major purpose of this study and Review of Relevant Literature is to examine syntax as it is relevant to narrative structure because previous research reported here does not relate syntax to narrative understanding.

\section{Relevance of Transitivity}

Linguists such as Givon (1984) and Hopper and Thompson $(1980,1984)$ have studied discourse and have also studied certain syntactic features as being discourse related. One such syntactic structure is transitivity. Transitivity refers to the syntactic and semantic processing of an entire clause where the action/event embodied in the verb is transferred from the subject to the object. The notion of transitivity is not a dichotomy, but rather a continuum that depends on the idea of prototypically where each feature of transitivity encompasses a different type of intensity as to how the action/event is transferred from the subject to the patient. Some examples of high transitivity are:

John hit Mary.

The dog bit Mary.

Action verbs hit and bit are easily transferred from the subjects, John and dog to the totally affected object, Mary.

Some examples of low transitivity are:

Wisdom is important.

Greed is bad. 
The copular verb, is does not transfer action from abstract subjects, wisdom and bad to a deleted object.

Hopper and Thompson (1980) focus on the notion of transitivity as a syntactic discourse feature. They suggested that transitive features are represented as global phenomena that are predominant in a wide variety of languages and thus are considered to be processes utilized by individuals in the construction of narrative discourse. Hopper and Thompson (1980) have identified the parameters of Transitivity that suggests a scale and is shown in Table 1:

Table 1

Transitivity Features According to Hopper and Thompson

High Transitivity

Low Transitivity

A. PARTICIPANTS

2 or more participants

1 participant

Agent and Object

B. KINESIS

action

non-action

C. ASPECT

telic

atelic

D. PUNCTUALITY

punctual

non-punctual

E. VOLITIONALITY

volitional

non-volitional

F. AFFIRMATION

affirmative

negative

G. MODE

realis

irrealis

H. AGENCY

A high in potency

A low in potency

I. AFFECTEDNESS OF O

O totally affected

$\mathrm{O}$ not affected

J. INDIVIDUATION OF O

$\mathrm{O}$ highly individuated

$\mathrm{O}$ non-individuated 
Each element of the Transitivity Scale includes a different component of the "effectiveness or intensity with which the action is transferred from one participant to another" (Hopper \& Thompson, 1980, p. 252). A complete explanation of each of the ten Transitivity features will follow:

Participants refers to the subject and object in a clause. All of the participants (subject and object) are needed to transfer the action event from subject to object. In order for a clause to contain a high degree of transitivity, both participants need to be animate and definite and the subject participant needs to be an agentive and the object needs to be totally affected by the action/event. Take the example:

Sam rescued the lady.

There are two participants in this clause and both are animate, Sam and lady. Sam is an agentive subject because he causes something to happen and is both animate and definite, lady is both an animate and definite object and is totally affected by the action/event, rescue.

Kinesis is embodied in the verb of a clause, and refers to an action and not a state where the action is transferred from participant to participant. For example:

Sam hugged the lady.

In this clause hugged has the kinetic/action quality where the action is transferred from Sam (agentive subject participant) to lady (object participant).

Aspect refers to verb action that has an end point(telic action). The telic action of a verb is more easily transferred from the subject/participant to the object/participant. For example:

Mary killed a lion.

The verb, killed has telic value, as the action has an end point and is easily transferred from the agentive/subject/ participant, Mary to the totally affected/ object/ participant, tiger.

Punctuality refers to actions that are carried out suddenly without a transitional phase between initiation and completion. A punctual verb has a more marked effect on the object 
participant than action verbs that are inherently ongoing; contrast hit (punctual) with carry (non-punctual). To carry an object has less of a marked impact than to hit an object. Take the example:

Sam hit the other player.

Hit is a punctual verb that in this case is considered to have a marked transition between inception and completion and the action of hit is transferred from the agentive/subject/ participant Sam to the affected/object/participant, player.

Volitionality refers to an agentive/subject/participant who carries out an action that has an apparent and direct effect upon the object/participant. For example:

Sam rescued the lady.

The subject/participant, Sam acted purposefully in order to execute the rescue. Contrast the previous example with, Sam forgot the lady. For Sam to forget the lady generally represents non-volitional motivation as it is considered in this example that the lady was not purposefully forgotten by Sam.

Affirmation refers to affirmative/negative parameters. An affirmative clause generally characterizes events that have taken place within a clause and a negative clause characterizes events that have not taken place. For example:

Sam rescued the lady

This is an affirmative clause as it is clear that, Sam rescued the lady. Contrast the negative clause, Sam did not rescue the lady. It is clear that this negative clause characterizes events that did not take place.

Mode refers to the distinction between realis and irrealis encoding of events. Irrealis refers to a hypothetical or non-real event. Realis refers to a real event that has occurred. For example:

Sam rescued the lady. 
This is an example of realis. This event, rescued is presented as a real event and not an hypothetical event in contrast to the following which is a hypothetical event:

If Sam had rescued the lady.

Agency refers to an animate/human /subject/participant that effects a transfer of the action/event to the object/participant as opposed to a subject/participant that is inanimate and does not posses its own agency. For example:

Sam rescued the lady.

Sam is a subject/participant that is human and effects a direct transfer of action/event, rescued to the object/ participant, lady. Contrast this example:

The lamp startled Sam.

Lamp in this clause is an non-human/ inanimate/ subject that lacks any of its own agency and therefore the event startled may be Sam's internal reaction or state.

Affectedness refers to features of the object/participant. Affectedness refers to the degree to which the action is transferred and effects the object.

Individuation, another feature of transitivity, refers to features of the object/participant. Individuation refers to the state of the object. The properties of individuation are as follows in Table 2. 
Table 2

Properties of Individuation

Individuated

Non-individuated

proper

common

human, animate

inanimate

concrete

abstract

singular

plural

count

mass

referential, definite

non-referential

Take the examples, Mary killed the tiger versus Mary killed some tigers. In both examples tiger is totally affected. In relation to individuation however, tiger in the first example is animate, concrete and referential while in the second example some tigers is less individuated as it is plural, mass and non-referential.

Since the ten features of transitivity have been described, it is important to understand how these ten syntactic features of transitivity represent a continuum rather than a dichotomy. A clause may have any number of the transitivity features. This research proposes that a clause that is highly transitive (high transitivity) will contain most of the transitive features while a less transitive clause (low in transitivity) will contain fewer (mass noun) transitive features.

\section{High Transitivity}

A clause that contains all ten of the transitivity features is considered to be highly transitive (Givon, 1984; Hopper \& Thompson, 1984). This research suggests that unconstrained summaries contain a greater percentage of highly transitive features in contrast 
to constrained summaries. Hopper and Thompson, in their 1984 article, propose that there are specific grammatical properties that characterize a highly transitive sentence. These properties include an action event that denotes a "concrete, kinetic, visible, effective action carried out by, and involving participants" (Hopper \& Thompson, 1984, p.707). Givon too indicates that there are three semantic dimensions which are central to high transitivity:

1. Agent: A highly transitive clause involves a volitional, controlling, initiating, active agent, one that is responsible for the event (Givon, 1984).

2. Patient (Object): The highly transitive event involves an inactive, nonvolitional, noncontrolling patient, one that registers the changes of state associated with the event (Givon, 1984)

3. Action Event (Verb): The highly transitive clause involves a compact (non-durative), bounded (non-lingering), non-hypothetical verb. It represents an event that is fast moving, completed and real (Givon, 1984).

The following is an example of a highly transitive clause because it contains all ten of the syntactic features of Transitivity:

$\begin{array}{lll}\text { Mary } & \text { killed } & \text { the tiger } \\ \text { +participant } & \text { +kinesis } & \text { +affectedness } \\ \text { +volitional } & \text { +telic } & \text { +individuation } \\ \text { +agency } & \text { +punctual } & \text { +participant } \\ \text { +individuation } & \text { +affirmative } & \\ & \text { +realis }\end{array}$

An application of Hopper and Thompson's (1980) scoring procedure for transitivity suggests that this clause contains all ten features of transitivity. Also, an examination of highly transitive features (Givon, 1984; Hopper \& Thompson, 1984) demonstrates that this clause represents a prototypically transitive clause. The subject/agent, Mary is volitional, controlling, animate, and initiating agent/participant who is responsible for the event/action, 
killed. The patient/object tiger, is individuated, inactive, nonvolitional, animate, noncontrolling object/participant that is totally affected by the action/event, killed. The action/event, killed is a kinetic, telic, punctual, affirmative, and realis event. An application of the works of Hopper and Thompson $(1980 ; 1984)$ and Givon (1984) support that Mary killed the tiger characterizes a highly transitive clause.

Hopper and Thompson (1980) conducted research with three narrative texts to investigate information in narratives. They determined that highly transitive features foreground discourse and contribute information about the sequenced main points of narrative discourse. In sum, their research demonstrated that highly transitive clauses contain the discourse feature of foregrounding.

\section{Foregrounding}

Foregrounding as a discourse function is to impart important ordered action sequences of the main points of discourse. Hopper and Thompson (1980) stated that "foregrounded portions of a narrative together comprise the backbone or skeleton of the text, forming its basic structure" (p.280) as is the function of narrative plot structure (Seifert, Dyer, \& Black, 1986; Thorndyke (1977). Polany-Bowditch (1976), in support of Hopper and Thompson (1980), contend that narrative plot structure and content incorporate aspects of foregrounding suggesting that narratives are comprised of "structure that presents temporal information that charts the progress of a narrative through time by presenting a series of events which occur sequentially" (p. 61).

In conclusion, the works of Givon (1984) and Hopper and Thompson $(1980,1984)$ have served thus far to develop a theoretical foundation and research base to support the notion that the syntactical features of high transitivity and foregrounded plot features are structures that can be used by readers during the production of summaries. Hopper and Thompson's (1980) research was an examination of the transitive narrative features themselves while the current research is an examination of the production of summaries 
through studying the transitivity features of the summaries. However, the use of low transitivity features in story summaries has not been addressed by Hopper and Thompson $(1980,1984)$, and this kind of summary is also a possible outcome of the current study.

For instance, examine this example of a constrained summary that is not high in transitivity. It does not have highly transitive features and does not contain foregrounded/plot structured narrative elements:

$\begin{array}{lll}\text { Wisdom } & \text { is } & \text { important } \\ \text {-agency } & \text {-telic } & \text { +subject complement } \\ \text {-volition } & \text {-kinetic } & \text {-affectedness } \\ & \text {-punctual } & \text {-individuation } \\ & \text { +affirmative } & \end{array}$

In this clause only one feature of transitivity is present (affirmative). Therefore, this clause is considered to be low in transitivity. This sentence does not impart any sequenced action events and is not highly transitive in nature. As determined by this researcher and an expert in the field of reading, this clause imparts abstract thematic information about that the story, "Fate".

The works of Hopper and Thompson $(1980,1984)$ provide an initial syntactic base for looking at summaries. Their work has begun to examine the differences between constrained summaries and unconstrained summaries. However, there is a need to examine other fields of study that help to explain the transitivity feature differences produced by both summaries.

\section{Relevant Cognitive Science Literature}

\section{Macrorules for Comprehension and Summary Writing}

From a discourse processing perspective, the works of Kintsch and van Dijk (1977) provide the cognitive base for looking at summaries. Their work lays the foundation for anticipated differences between constrained and unconstrained summaries. The purpose of this section is to present research into comprehension and the summary-writing process that help 
account for the syntactic and story feature differences anticipated in both the constrained and unconstrained summaries. Further, this research to be reviewed supports the observations (Brown \& Day, 1983; Marshall, 1984, 1994) that features of Kintsch and van Dijk's (1977) model are production processes used by readers during the production of both summary types. The work of Kintsch ignores syntax in general as related to summary writing, summary production, and narrative understanding while this study examines syntax as it relates to narrative structure and summary production.

Kintsch and van Dijk's (1977) macrorules for comprehension and summary writing and the works of Brown and Day (1983) and Marshall (1984) provide the research framework that characterizes certain process differences in production patterns for both of the kinds of summaries. Initially, an overview of the summary and comprehension production model operation will be presented. This will be followed by a detailed explanation of the macrorules. Next, possible process explanations are presented of how the integration and generalization macrorule may be applied by the reader during the production of an unconstrained summary to produce highly transitive features and how the construction macrorule may be applied by the reader during a constrained summary to produce low in transitivity features.

\section{Summary and Comprehension Production Model Operation}

Kintsch and van Dijk (1977) developed a process model of second order discourse representation. Kintsch and van Dijk's (1977) model is concerned with the production of second order discourse, or discourse with respect to another discourse such as a summary. Kintsch and van Dijk's (1977) goal was to describe the "system of mental operations that underlie the processes occurring in text comprehension and in the production of recall and summary protocols" (p. 363). The model is comprised of an input list of propositions that represent original text meaning. The propositions are coherently ordered in the original text base. The reader proceeds through the whole text, constructing cyclic networks of coherent discourse. Each processing cycle has a certain number " $n$ " of original text propositions. The 
propositions of original text are processed by the reader as the reader's schemata classifies all of the propositions of the original text base.

During the production of second order discourse the macrorule-operators (deletion, generalization, integration, and construction) transform the propositions of the original text into a set of propositions that represent the meaning of the text. The reader during the production of second order discourse for example, deletes, generalizes, integrates or constructs propositions from the original text, and in the second order discourse certain propositions are reproduced or invented. These are explained in the next section.

The Macrorules

According to Kintsch and van Dijk (1977) the semantic structure of discourse is represented at two levels, the levels of microstructure and the macrostructure. The microstructure refers to the structure of the individual propositions and their relations while the macrostructure refers to the global aspects of the discourse as a whole. This theory suggests that readers process multiple layers of meaning simultaneously and can use these multiple layers to produce different recalls depending on the task. Thus constrained summaries, due to the length constraints, are the product of a different set of macrostructure elements than used for the unconstrained summaries. In fact, it is anticipated that constrained summaries produce use of the construction macrorule while unconstrained summaries produce use of the integration and generalization macrorule. The constrained summaries will contain little or none of the microstructure due to the restrictions placed on the recall condition while the unconstrained summaries will contain both macrostructure and microstructure elements.

The macrorules include the following levels of processing text for comprehension and summary:

1. Deletion - unimportant or redundant facts of text are deleted

2. Generalization - general statements are made in response to 
actions and nouns and involve the substitution of a subordinate, For example : lilac, tulip, rose = FLOWERS (subordinate)

3. Integration - integration of ideas. For example: 1 . I got a ticket. 2 . I brought my luggage to the trains. etc. $=$ GOING ON A TRIP.

4. Construction - refers to the summary of main text concepts of a text through the construction or inclusion of ideas that are not included in the text. A major concept of the text is produced as a topic sentence or if a suitable topic sentence is not present the reader invents a suitable topic sentence. The construction macrorule produces generalized text recall while the integration and generalization macrorule produce more specific text recall.

The Works of Brown and Day and Marshall

Kintsch and van Dijk's (1977) model was supported by the empirical research of Brown and Day (1983) and Marshall $(1984,1994)$. It has been demonstrated that in the production of a unconstrained summary older readers have a greater likelihood to apply the generalization and integration macrorules (Brown and Day, 1983) while in the production of a constrained summary older readers have a greater likelihood to apply the construction rule (Marshall, 1984, 1994).

Brown and Day (1983) conducted developmental research to examine the use of the macrorules for paraphrasing expository tests through a series of three experiments. In experiment I, results indicated that high school students and college students used the macrorules of generalization and integration, in contrast to the fifth and seventh graders who used a deletion strategy to construct their summaries. In experiment II college rhetoric teacher outperformed college freshmen in the ability to utilize the construction strategy to produce summaries. In the experiment III junior college students performed on the level of seventh graders who relied less on the deletion strategy and more on the construction integration strategy when producing summaries of text. Brown and Day's (1983) research provided the empirical evidence to support Kintsch and van Dijk's (1977) summary model. In 
summation, younger subjects tended to use the deletion macrorules while older subjects tended to use the generalization and integration macrorule and experts used the construction macrorule. The works of Brown and Day (1983) support the theoretical notion that older readers' application of the generalization and integration macrorule is task dependent upon use of a unconstrained summary as a means of representing narrative understanding.

Marshall $(1984,1994)$ also conducted developmental research to examine narrative summary strategies used by fourth, sixth, eighth, eleventh graders and adults. The subjects wrote constrained rather than unconstrained summaries. Results indicated that the fourth graders utilized the deletion strategy, sixth graders utilized mostly the generalization strategy, eighth graders utilized features of deletion and generalization, the eleventh graders utilized features of generalization and construction and the adults utilized features of generalization and construction. The differences between the results of Brown and Day (1983) and of Marshall $(1984,1994)$ might be attributable to the difference in the length of summaries. This would support the theoretical notion that older readers' application of the construction rule is task dependent upon use of a constrained summary as a means of representing narrative understanding.

The works of Brown and Day (1983) and Marshall $(1984,1994)$ have supported the validity of Kintsch and van Dijk's (1977) macrorules for comprehension and summary-writing production. The work of Brown and Day (1983) addressed the production patterns produced in older readers' unconstrained summaries while the work of Marshall addressed the production patterns produced in both younger and older readers' constrained summaries. However, there is a need for further research that directly examines narrative summary writing production differences produced in a unconstrained summary and a constrained summary and to explore more directly the relationship between task constraints and summary content. Since readers access both semantic and syntactic information while reading and writing it is important to explore the interaction of both kinds of information. Following is an explanation 
of how the application of the macrorules is task dependent and contains varying degrees of transitivity.

Synthesis of Macrorules and Transitivity

Production of an unconstrained summary has definite production differences from a constrained summary. Kintsch and van Dijk (1977) describe that in the production of an unconstrained summary certain syntactic features are prevalent. These features include the use of action verbs and concrete nouns that involve a substitution of a subordinate (generalization rule) or use of action verbs and concrete nouns that involve the integration of ideas (integration rule). Their argument is consistent with the concept of transitivity as a marker for unconstrained summaries. In this current study it was hypothesized that unconstrained summaries produced by older readers contain a greater percentage of highly transitive discourse features. Highly transitive features include an action/event embodied in a verb that is transferred from a concrete subject to a concrete object (Hopper \& Thompson, $1980,1984)$. The macrorules of integration and generalization as detailed also involve propositions that contain action/events embodied in verbs and concrete nouns and the integration of ideas (Kintsch \& van Dijk, 1977). For instance:

Sam rescued Manny.

Rescued is an action verb that is easily transferred from an agentive concrete subject, Sam to a concrete totally affected direct object, Manny. Further, this statement represents the use of concrete nouns and verb to integrate and generalize ideas.

More specifically, unconstrained summaries are produced through an application of the macrorules of generalization and integration and the integration and generalization macrorules are actualized through concrete/action event propositions from the original text. From a syntactic perspective, an application of the generalization and integration macrorules represent the syntactic and discourse features of high transitivity. 
Production of a constrained summary, due to its length constraints, has a definite task dependent production differences from an unconstrained summary. Because only one sentence can be written the writer is forced to focus less on the microstructure of the text than does an unconstrained summary. Kintsch and van Dijk (1977) suggest that the application of the construction macrorule produces a topic sentence that is accessed by abstract text schemata and represents global understanding of a text that includes the readers' opinions and personal knowledge. For the current study, it was hypothesized that a constrained summary produced by older readers would contain a greater percentage of low in transitivity features and will be the result of the application of the construction macrorule. The construction macrorule involves propositions that represent the decontextualized topic sentence macrostructure of text. The works of Marshall $(1984,1994)$ support the contention that constrained summaries are produced by older readers through the application of the construction macrorule and contain propositions that are invented and not included in the original text. These summaries appear to represent abstract schema rather than concrete event based schema. For instance, Spiritual things are more important than superficial is an example of a constrained summary. This example represents the application of the construction macrorule, abstract understanding of a story that contains low transitivity features, has been invented, and is not contained in the original text.

In conclusion, the works of Kintsch and van Dijk (1977), Brown and Day (1983), and Marshall $(1984,1994)$ provide the theoretical and empirical evidence that suggests that the length differences between the constrained summary and the unconstrained summary create differences where the older reader applies different sets of macrorules. However, these works ignore syntax in general while this study examines transitivity as one example of how syntax is relevant to narrative understanding and narrative summary production. The form of the constrained summary forces severe coordination of information while simultaneously preventing superficial reader response. The integration and generalization macrorules are 
applied to produce a unconstrained summary that activates propositions that are high in transitivity (action verbs and concrete nouns) and are accessed through concrete/action schemata while the construction macrorule is applied to produce a constrained summary that activates propositions that are low in transitivity and represents in a topic sentence the macrostructure of text comprehension and is accessed through abstract schemata.

\section{Relevant Theories of Narrative Literature}

\section{Story Grammars}

The purpose of this section is to review the literature related to the features of story structure and the way in which people rely on story structure when comprehending. The works of Thorndyke (1977) showed that a generic plot-structure of story is essential in building meaning for specific stories. The typical plot structure of a story has become known as story grammar.

Story grammars are one of the predominant theories of narration in cognitive science and have provided a foundation for much empirical research conducted during the last two decades. Story grammars are a set of rules that purport to generalize to all stories from Western European cultures and capture all of the structural properties of a story. In fact, our expectation that a story will conform to the patterns described by a story grammar are so robust that we sometimes change the meaning and content of stories that are not highly generalized to make them fit the prototype (Bartlett, 1932).

Most story grammars are based upon several assumptions. The first is that story material has a kind of syntax much like sentences. The second assumption is that stories are comprehended and organized in terms of hierarchical networks of categories and the logical relations that exist between these categories.

Stein and Glenn (1979) contended that story grammars generally include: (1) story, (2) setting, (3) episode system, (4) initiating event, (5) response, (6) internal response, (7) plan 
sequence, (8) internal plan, (9) plan application, (10) attempt, (11) resolution, (12) direct consequences, and (13) reaction. The organization is shown graphically in Figure 1.

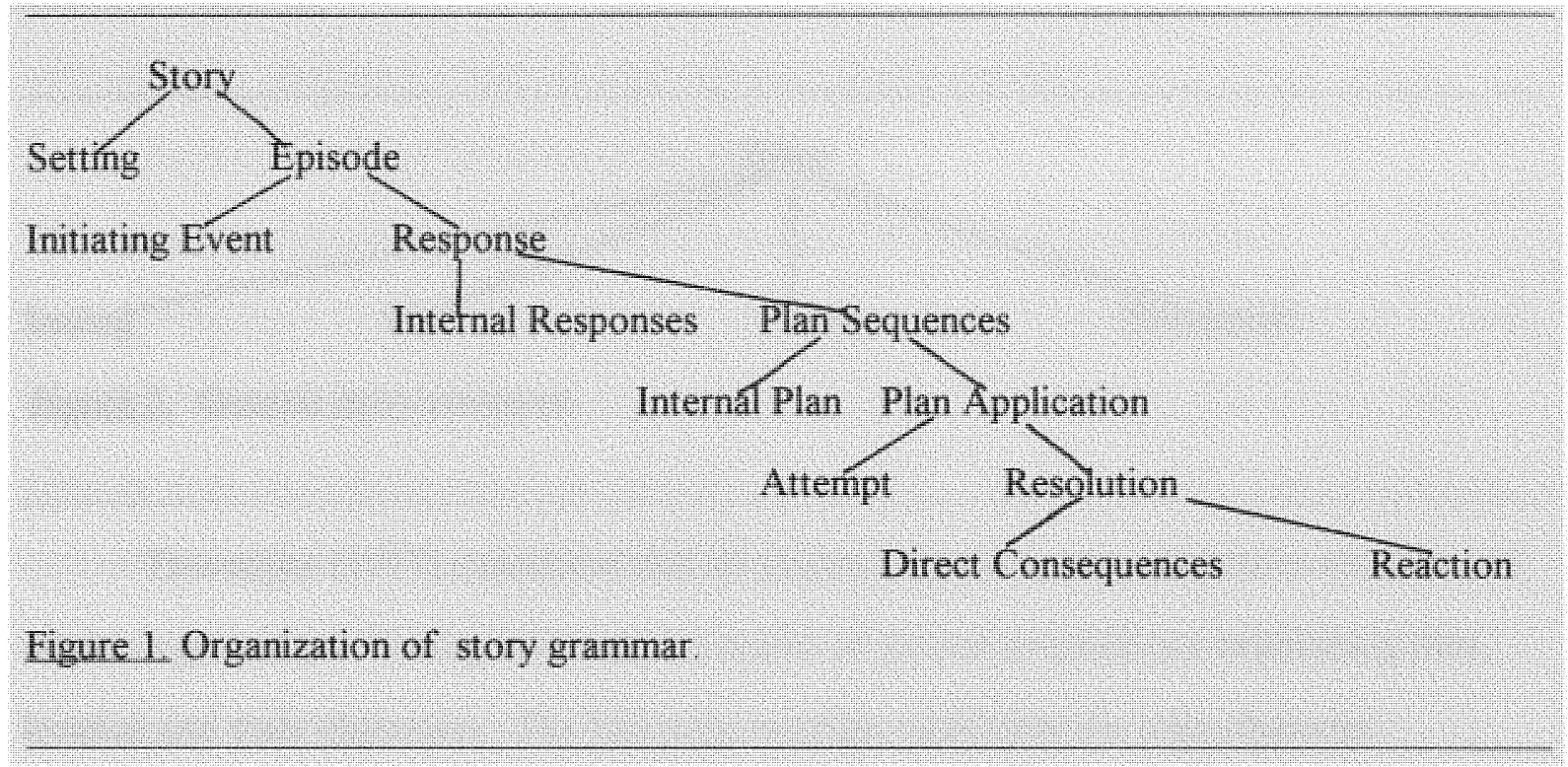

Stein and Glen (1979) conducted developmental research with children to validate a schema for stories. They found that children as young as five both produced and recalled stories using this structural sequence. Their research did answer some fundamental questions about children's organization of story information. A limitation of their study was that there was little emphasis on story content, no emphasis on theme as a feature of story structure and no emphasis on variations between story schemata activation.

The early works of Thorndyke (1977) began to examine theme as an organizational feature of plot. Thorndyke (1977) examined the structure and content variables on comprehension and memory of simple narratives. The focus of his work was to examine the level of story structure that constitutes a story schema and is common to all simple narratives. His research demonstrated that generic story structures are used by subjects during the story comprehension process and that story content and structure play a role for comprehension and memory of simple stories. 
Thorndyke's (1977) research lead to the development of the following generic story structures: (1) setting, (2) theme, (3) plot and (4) resolution. The organization is graphically shown in Figure 2.

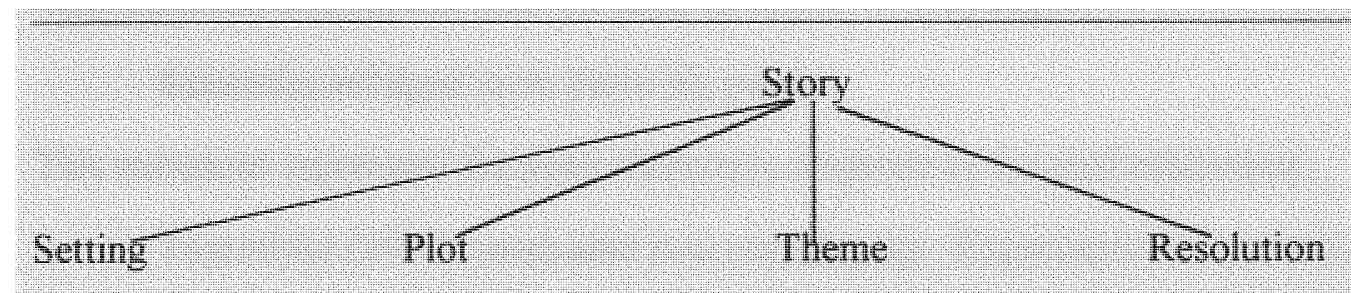

\section{Figure 2. Generic story structure.}

Thorndyke (1977) considered that all aspects of generic story structures are elements of plot organization. Setting information consists of several propositions establishing time, location and main characters. The following example establishes the main character:

Sam was nearly twenty-four, almost good-looking and quite penniless. The following example establishes time and location:

Samantha had already arranged in her mind the lunch she should give at her house in Curzon Street.

Theme of a story is the main focus of the plot and is the justification and introduction of a goal of the main character and the sequence of events that lead up to its realization. For example:

It was Samantha's pleasure and intention that she should have a party. This type of statement is an example of the general focus to which a plot adheres as well as includes the stated goal of the main character. The plot of a story is any number of episodes comprised of action attempts to achieve the main characters goal. For example:

Sam helped the woman in a difficult situation.

He is able to rescue her. 
This represents an action of the main character. Resolution is a statement of the final results of the story that is connected to theme. Resolution is comprised of successful goal attainment of the main character or the main characters response to the goal attainment that is consistent with character satisfaction with the goal outcome. For example:

Samantha indulges in no more big parties.

This statement represents the main characters response to the goal attainment.

Thorndyke (1977) conducted two experiments. Experiment I was conducted to assess the effect of changing the amount of plot structure on a reader's memory for a story. The subjects were 64 undergraduates at Stanford University. Two stories were utilized for this experiment with structure ranging from highly stereotypical narrative form to a structure with little narrative structure. The original passages were presented either visually or auditorally to the subjects. Subjects were asked to give a complete written recall of the passage after the first passage was heard, give a short written summary and complete a recognition test after each passage was presented. For scoring, the recall protocols were segmented into propositions. Each passage contained 35 propositions. A proposition was determined to be a clause or a sentence which contained an action or stative verb. The protocols were scored for gist recall of propositions. The passage summaries were also scored to determine their length and propositional content. The scoring criteria was identical to those used for recall. The results presented after the data had been collapsed across both modes of presentation supported the importance of a well-learned plot organization structure for comprehension and memory of story.

Thorndyke (1977) conducted a second experiment because of the subjects' confounding of structure and content differences between the two stories in Experiment I. The subjects consisted of forty-eight Stanford University undergraduates. In Experiment II the plot structure and passage content were varied orthogonally to determine their separate effects on recall. The results suggested that both structure and content play a role in memory 
for simple stories. In both experiments story comprehension and recall were correlated and found to be a function of the amount of identifiable plot structures in the passage.

Thorndyke's (1977) research demonstrated that memory for stories tends to include organizational story elements such as setting, theme, plot, and resolution which are all representations of plot organization.

Thorndyke's (1977) model for simple narrative structure does provide a foundation for simple story comprehension produced through unconstrained summaries. However, this model does not account for the following: (a) a more sophisticated class of narratives, (b) abstract theme that takes on a more societal or universal understanding story comprehension, (c) how plot organization and abstract theme organization are reflected syntactically. The major limitation of Thorndyke's (1977) work is that there is a need to examine theme that is not schema represented by action/event oriented plot features but represented by abstract schema features.

The research of Brewer and Lichtenstein's (1980) into structural affect theory, Rosenblatt's (1986) reader response theory, and Many's (1991) reader response stances begin to examine the differences between summary types. Also the works of Schank (1982) and Seifert, Dyer and Black (1986) begin to examine the thematic level of knowledge representations as elements of abstract story understanding that are produced by constrained summaries.

The work of Brewer and Lichtenstein (1980) acknowledges the important role of reader affect in the narrative comprehension process and begins to account for syntactic features and schemata differences between both summary types. Primarily, structural affect suggests that plot features provoke readers emotion and therefore are more memorable.

This research suggests that structural affect theory begins to examine a reader's affect organized schema that is evoked during the production of an unconstrained summary. Such a summary is high in transitivity and represents plot features. 
Brewer and Lichtenstein (1980) conducted research to test the structural affect approach to story organization. They developed narratives and manipulated the basic discourse organization. Subjects were asked to read the narratives and were stopped at fixed points while reading and asked to make affect judgments. Results indicated that story plot features that require emotional response are more memorable to readers.

The work of Brewer and Lichtenstein (1980) provides a cognitive affect base for examining narrative comprehension that represent affect schema that is highly memorable and produced through an unconstrained summary. Structural affect theory represents a specific comprehension affect schema that accounts for high in transitivity features that are anticipated to be produced by an unconstrained summary and begins to account for memory differences between both types of summary.

The work of Rosenblatt (1986) provides another cognitive affect schema base for examining constrained summary and unconstrained summary differences. Reader response theory emphasizes the reader's role during the reading of a literary work. According to Rosenblatt (1986) the reader's role during the narrative comprehension process elevates the reader to equal status with the text. The text provides ideas, imagery, and feelings during the comprehension process. Thus, narrative comprehension becomes the convergence of the reader and the text.

Rosenblatt (1986) suggests that narrative comprehension involves the reader acquiring a stance or schema that indicates the readers focus of attention during the comprehension process. There are two types of stances, an efferent stance and an aesthetic stance. An efferent stance indicates that the reader is directing her attention toward less superficial generic or universal information generated by the text while an aesthetic stance indicates that the reader is directing her attention toward more superficial affect associations generated by the text. Rosenblatt suggests that when reading literature the aesthetic stance is evoked and while reading expository text the efferent stance is evoked. The differences between both of 
these stances begins to provide evidence that begins to account for differences between both summary types.

Many (1991) conducted research that demonstrated that when reading literature both the aesthetic and efferent stances are evoked. The subjects for this research included 43 fourth graders, 47 sixth graders, and 40 eighth graders. Subjects all read the same three short stories and completed written unconstrained summaries. The responses were examined for reader stance and level of understanding. Level of understanding refers to whether the reader understood the text from a text explicit level to a text implicit level. Results of this research revealed that use of an aesthetic stance was associated with more implicit levels of understanding while there was no significant main effect for efferent stance and level of understanding. However, efferent stance was produced by use of some unconstrained summaries. This research provides a base of investigation for efferent stance and what type of summary task would consistently elicit the efferent stance. Efferent stance involves the reader directing attention toward universal information generated between the reader and the text. A universal understanding of text is generated in a constrained summary. Thus, reader stance begins to account for some differences between constrained and unconstrained summaries.

In sum, the work of Stein and Glenn (1979) provides evidence to support the idea that story grammars are generic cognitive story comprehension structure that generalize to all stories. The work of Thorndyke (1977) begins to examine theme as a story comprehension structure that generalizes to all simple stories. Thus, far explanation for plot organization that is represented in unconstrained summaries has been demonstrated. However, the type of story structure produced in constrained summaries has not been addressed. The works of Brewer and Lichtenstein (1980), Many (1991), and Rosenblatt (1986) begins to explain some of the syntactic, memory, and story feature differences between a constrained and unconstrained summary. This research suggests that an unconstrained summary due to its length forces 
specific coordination of information while producing more superficial highly transitive, action/event responses that are produced by the reader's use of an aesthetic/affect stance or schema. A constrained summary due to its length constraint forces coordination of information while simultaneously preventing very superficial, high in transitivity, action/event responses rather producing affect responses that are produced by the reader's use of an efferent stance or schema.

Relevant Artificial Intelligence Literature

\section{Concrete Schemata}

The initial work in the area of memory structure revolved around schema theory (e.g. Bartlett, 1932). Schemata organize inferences in memory and serve to connect related information in networks. Schemata can organize any type of information from pictures to affective experiences and are built upon our world experiences. For example an event sequence like, "going to a restaurant" can be organized in memory as a schema containing events, setting information, etc. This type of memory organization can provide predictions for the events that are about to occur and explanation if they do not occur. Thus, concrete schemata organize all types of experiences from how to play baseball to the way to negotiate (Schank \& Abelson, 1977).

Scripts represent a subset of concrete schemata. Scripts are the schemata used to understand or construct a story. Schank and Abelson (1977) have programmed scripts into computers and made it possible for the computer to predict correctly the next sequence of events. They contend that people also use scripts to anticipate their responses and the behavior of others. Scripts provide generic concrete knowledge structures that correlate to frequently enacted activities. Take for example the 'restaurant' script. There are roles (customers), props (utensils), entry conditions (hunger), results (being full), and configurations of actions that are segregated into scenes (e.g. enter the restaurant, order your food, etc.). Scripts are generic knowledge structures that represent stereotypical sequences 
that unfold in a particular order and when a narrative violates the script, a reader will often "recall" a normal sequence rather than the atypical sequence.

When a particular content script is triggered during comprehension the script knowledge guides the course of text understanding. Scripts too, provide inferences and expectations by default. Even if the text leaves information out, the script will enable the reader to fill in the missing information. For instance, in relation to the baseball script the text does not have to give all of the information about playing baseball but the reader can still envision the game. Script structure is useful in encoding organizations of knowledge that are relatively stereotypical however, they fail to capitalize on similarities between scripts. For example both in a football script and in a baseball script you play a game. However, research has demonstrated that similarities between scenes with a script introduced subject recognition problems (Seifert, Dyer, \& Black 1986). Therefore, scripts, account for only one kind of limited information presented in narratives, and there is a need to account for memory structures that capture similarities between scripts. This led to the development of Memory Organization Packets (MOPs) (Schank, 1982).

MOPs organize similar information as scripts do, but they allow scenes to function as independent entities in memory (Schank and Seifert et al, 1986). MOPS represent independent concrete memory structures to be accessed during comprehension (Schank, 1982) as plot structure represents independent concrete generic memory structures utilized during the comprehension of any number of narratives (Thorndyke, 1977). MOP structures allow new information available in any similar situation to be accessed. For instance, a game playing script is stored with all game playing scripts which makes the information available in any game playing situations. Now, there is a generalization of common subparts of scripts which allows greater flexibility rather than general stereotypical whole script activation.

MOPs generalize information on the basis of similarities of episodes and plot features generalize story comprehension on the basis of order action events that describe the main 
points of a story. Thus, it is that production of a unconstrained summary that produces plot features for narrative representation. MOPs generalize narrative comprehension on the basis of ordered action events. This begins to explain how during the production of a unconstrained summary the reader applies the generalization and integration macrorule which emphasizes concrete nouns and action verb clauses from the original text and produces plot features that contain highly transitive features. However, MOPs function to examine stereotypical concrete action schemata rather than abstract schemata.

A schema for abstract comprehension representations is still needed to explain how an application of the macrorule of construction produces a constrained summary that contains low in transitivity features that is primarily nonplot organized.

Abstract Schemata

The purpose of thematic level of knowledge is to begin to extract story information that makes a distinction between specific story features represented by plot and generic story features represented by abstract themes. According to Seifert, Dyer, and Black (1986) some of our world knowledge is derived from circumstances of an episode while others lie in a more abstract representations of the relations between concepts in an episode.

Schank (1982), who was working in the area of artificial intelligence story comprehension, began to explore thematic information that is abstract and not dependent on contextual features. Schank (1982) proposed that thematic structures attempt to characterize story comprehension abstract and complex patterns of goals and plans that are not entirely action based. Schank's data indicated the content of potential thematic story comprehension memory structures. These story structures are referred to as, Thematic Organization Points (TOPs).

TOPs represent problems occurring during the pursuit of a goal and the issues relating to what happens after a goal succeeds or fails (Schank, 1982). Take this example: 
$\mathrm{X}$ was talking about how there were no oranges around for a month or two. Than all of a sudden everyone was able to get as much as they wanted. But the price had gone up 25 percent. This reminded $\mathrm{X}$ of the oil situation the previous year. We were made to wait on lines because of a shortage that cleared up as soon as the price had risen a significant amount.

This thematic organization point (TOP) in memory is built around the conditions that affect the planning for the possession goal (Schank, 1982). The conclusion included in this structure is that unethical people who control a commodity will put it back on the market when the price has risen.

TOPs as knowledge structures (Schank, 1982) are involved in a variety of memory tasks. These include the ability to:

1. Get reminded of a story that illustrates a point.

2. Come up with adages such as "Every cloud has a silver lining" at an appropriate point.

3. Recognize an old story in new trappings.

4. Notice co-occurrences of seemingly unrelated events and draw conclusions.

5. Know how something will turn out because the steps leading up to it have been seen before.

6. Learn information in one situation that will apply in another.

7. Predict an outcome for a newly encountered situation.

TOPs as abstract knowledge schemata provide a wide range of memory tasks and need more concise empirical observation of each of the described memory tasks.

\section{Thematic Abstract Units}

Schank (1982) suggested examining the similarities between common themes that are revealed when one is reminded of thematically similar episodes. This is based upon the patterns of goal and plan interactions that people recognize and remind people of other 
experiences, captured in adage like sayings. Thus, an adage is an effective way of characterizing the theme of an episode (Seifert, Dyer, \& Black, 1986). Specifically TAUs focus upon planning failure that frequently occur (Seifert, Dyer, \& Black, 1986) in narration.

Seifert, Dyer, and Black (1986) began to investigate thematic patterns across episodes that vary in some respects and share more abstract thematic similarities. Specifically, thematic patterns provide a level of information that is useful in building memory structures. Thematic patterns store episodes in memory and provide the connections to related world experiences From a memory standpoint, plot, which is action sequenced events, is accessed by concrete schemata while thematic abstract units are the product of accessing abstract schemata.

According to Seifert, Dyer, and Black (1986) initially information at the plot level are episodic memory structures that are needed to access appropriate thematic structures in memory. TAUs represent abstract representations of plot organized story comprehension (Seifert, Dyer, \& Black, 1986). Episodic memory structures organize events involving similar kinds of planning failures. A planning failure refers to when something goes wrong for a character. Thus, TAUs are designed to be used as warnings in planning, while plot units do not incorporate that look-ahead goal into building a representation. Certain narratives contain similar TAUs that characterize an abstract comprehension representation (Seifert, Dyer, \& Black, 1986). TAU structures are important both within a story and as a relation between stories. TAUs serve to represent thematic/abstract structure of episodes.

Seifert, Dyer, and Black (1986) conducted two experiments to investigate whether subjects were able to understand, reproduce and make similarity judgments as predicted by TAU structures. In the first experiment fifty-six Yale undergraduates were participants. The subjects were given three example stories to read based on TAUs suggested by Dyer (1983) and were asked to write new stories based on the three examples. The subject generated stories were scored for which TAU pattern was in the story. Overall the TAUs, $82 \%$ of the 
stories clearly matched a TAU. Generally the subjects were able to recognize and reproduce a TAU.

In the second experiment thirty-six Yale undergraduates were participants. In this experiment, the relationship between stories based on TAUs was examined using a clustering methodology to investigate story structure. The stories were based on six TAU groups:

1. Hypocrisy, pot calling the kettle black

2. Incompetent-advice, the blind leading the blind

3. Unsupported Plan, counting chickens before they are hatched

4. Backfires, cutting off your nose to spite your face

5. Acting Too Late, closing the barn door after the horse is gone

6. Self-Deception, hiding your head in shame

A hierarchical clustering analysis revealed how strongly pairs of stories were related. Results indicated that of a total of 261 groups, 250 were appropriately labeled. An analysis of labels subjects used to describe the groups they formed revealed a high degree of agreement even in the lexical items used. Most of the labels were accurate descriptions of the TAUs premises and some coined new phrases. Results from both experiments indicated that subjects are sensitive to thematic patterns and are able to use thematic information in judgment of similarity.

In conclusion, these experiments indicated that TAUs do represent important thematic information about stories and that subjects are able to use this information to perform particular tasks. The thematic level of knowledge appears to be captured by structures that contain relatively abstract information about goal and plan relationships. Thematic knowledge is needed to understand points of episodes. The premise that thematic structures are useful in encoding and organizing related episodes suggest interesting properties of memory. Further experimentation on the representation and the specific processing functions of thematic knowledge structures will serve to determine the nature of their role in understanding. 
In the Seifert, Dyer and Black (1986) research in the first experiment the subjects read TAU based stories and were asked to write new stories based on the adages, and in the second experiment the subjects were asked to label stories according to six TAU groups. Therefore, the products revealed from the Seifert, Dyer and Black (1986) research focused on reproductions and labels that are directly correlated to specific TAUs. They did not attempt to find out whether readers can generate their own TAUs spontaneously. The current study is designed, in part to find this out.

\section{Multidimensional Theory of Narrative Comprehension}

The Review of Relevant Literature has presented information to support the development of a multidimensional theory of narrative comprehension. However, each element reviewed here is incomplete by itself. Narrative comprehension is a complex process and multiple schemata are used to make sense of a text. Previous research has looked at some elements of narrative comprehension, but has ignored its complexities.

First, the works of Givon (1984) and Hopper and Thompson (1980; 1984) provide the background to examine narrative discourse from a syntactic perspective. They have looked at high and low transitivity features in discourse. Their work however, has not investigated how transitive features are produced as features of narrative summary writing comprehension. Second, the work of Kintsch and van Dijk (1977) provide a cognitive discourse processing base for looking at summaries. Their work begins to examine discourse processing an unconstrained summary. However, their work does not examine the differences between a constrained summary and an unconstrained summary nor does it examine the syntactic features associated with summary writing. Third, the works of Brown and Day (1983) and Marshall (1984) have supported the validity of Kintsch and van Dijk's (1977) macrorules for comprehension and summary-writing production. However, their work does not compare the syntactic features and neither research examines narrative comprehension differences between constrained summaries and unconstrained summaries. Fourth, the work of Thorndyke (1977) 
began to develop generic story structures. His early works examined the organizational feature of plot and content related theme. Thorndyke's (1977) work did not examine universal thematic organization nor did his work address syntactic feature differences and summary production features related to narrative comprehension constrained summaries and unconstrained summaries. Fifth, the work of Brewer and Lichtenstein (1980) began to examine narrative comprehension that is related to structural affect theory where narrative comprehension is attributable to emotional responses evoked by the reader during the comprehension process. This work began to provide some answers about the syntactic and story feature differences between both summary types. Sixth, the works of Rosenblatt (1986) and Many (1991) began to examine narrative comprehension that is related to reader response theory where the reader uses either an efferent schema or an aesthetic schema to evoke narrative comprehension. The works of Rosenblatt (1986) and Many (1991) did not examine syntactic features, summary type production differences or story feature differences between constrained and unconstrained summaries. Direct investigation of these properties of comprehension is needed. Seventh, the work of Schank (1982) began to examine narrative schema and comprehension that is related to script theory, MOPs, and TOPs. Schank's (1982) work began to provide answers about schemata that are used to produce plot organized narrative comprehension. However, his work did not investigate syntactic features and summary differences that are produced by use of script schema, MOPs schema, and TOPs schema. Finally the works of Seifert, Dyer, and Black (1986) examine thematic abstract units (TAUs) that are related to narrative comprehension. Their work did not investigate whether a constrained or unconstrained summary produces TAUs. This research is designed, in part to find out whether readers can generate their own TAUs spontaneously.

The research conducted here examines the complex nature of narrative comprehension and has begin to develop a multidimensional theory of narrative comprehension from the works of others in the field of narrative understanding. 
The research reported here specifically examines comprehension differences that are produced in a constrained summary and an unconstrained summary. Thus, the multidimensional theory of narration proposes that readers have access to multiple semantic and syntactic strategies that are used in summary production.

Summary is a type of reader recall of a narrative. Recall has for this research two dimensions. Recall refers to either concrete representation of narrative comprehension or abstract representation of narrative understanding. It is of importance at this point to recognize the inherent difference between comprehension of narratives from a plot or concrete perspective and an abstract thematic perspective. Plot or concrete understanding is bound to the action/event attributes of the narrative while abstract thematic understanding is bound to a more universal and/or societal understanding of narratives. 
Chapter III

Methodology

The present study was designed to test the hypothesis that a constrained summary and a unconstrained summary would produce differences in the readers' use of story structure features and in the use of transitivity features and to develop a multidimensional theory of narrative comprehension. The previous discussion of literature has looked at certain elements of narrative comprehension but has ignored its complexities. This study was designed to examine the complex nature of narrative comprehension by examining how readers use different schemata simultaneously for comprehension. In particular, this study demonstrated that readers use task dependent schemata during the narrative comprehension process. More specifically, it is hypothesized that the constrained summary would produce thematic abstract unit story features that contain low in transitivity features while a unconstrained summary would produce plot oriented story features that contain highly transitive features.

The multidimensional theory of narrative comprehension proposes that a constrained summary, due to its, limited length, forces severe coordination of information while simultaneously preventing a reader's superficial response to narratives. During the production of a constrained summary the reader uses specific semantic strategies and syntactic strategies. The theory also proposes that an unconstrained summary, due to its length forces specific coordination of information while simultaneously producing more superficial response to narratives. Therefore, during the production of an unconstrained summary the reader uses different semantic strategies and syntactic strategies. Each type of summary provides different ways of representing narrative meaning.

\section{Hypotheses}

The specific hypotheses being tested in this study are listed below that propose that differences in both summary types are due to length. 


\section{Null Hypotheses}

1. There is no difference between the mean number of transitivity features used in a constrained summary and an unconstrained summary.

2. There is no difference between the proportion of usage of plot in a constrained summary and an unconstrained summary.

3. There is no difference between the proportion of usage of thematic abstract units in a constrained summary and an unconstrained summary.

4. There is no difference between the proportion of usage of theme in a constrained summary and an unconstrained summary.

\section{Research Hypotheses}

1. An unconstrained summary will contain predominantly plot features because of its length.

2. An unconstrained summary will be written using highly transitive features thus reflecting action orientation of summary because of its length.

3. A constrained summary will contain predominantly thematic abstract units because of the constraints on its length.

4. A constrained summary will be written using low transitivity features thus reflecting more stative and copular verbs because of the constraints on its length. Design

In an experimental study such as this one, the researcher manipulates an independent variable, controls for other extraneous variables, and examines the effect of the independent variables upon the dependent variables. For the purposes of this study there is one independent variable, summary type: a constrained summary containing a single sentence of from five to 15 words and an unconstrained summary of at least three sentences and no other length limit. The reason for the limits on the constrained summary, according to Marshall (1984), is to create a situation in which readers cannot respond with a one or two-word label 
or with a detailed retelling. The reason for requiring at least three sentences in the unconstrained summary is to create a situation in which the reader must create a response that is different in length and, hopefully, in content because of the greater length. The theoretical framework for this contention was fully discussed in Chapter II of this dissertation and suggests that the different length constraints between summaries produce differences in story structure elements recalled and number of transitivity features used. An unconstrained summary forces specific coordination of information while simultaneously producing more specific superficial response which represents high in transitivity, plot features of narrative meaning. A constrained summary forces severe coordination of information while simultaneously preventing superficial response that is represented by low in transitivity, thematic abstract unit features.

The four dependent variables for this study include the story structure elements of plot, theme, thematic abstract units and the syntactic feature of transitivity. These dependent variables are measurable and are examined as to how they are contained in both of the summary forms. For this study the dependent variables of story structure features are dichotomous measures. A dichotomous scale classifies data into two categories. A response is either present or not present. Either the summary recall is measured as either having a particular story structure element or is identified as not having a story structure element.

The measurement of the story features was developed from the works of Seifert, Dyer, and Black (1986) and Thorndyke (1977). The work of Thorndyke (1977) identified the following story features: setting, plot, resolution, and theme. For the purposes of this research setting and resolution were collapsed to create one plot category because according to Thorndyke (1977) setting and resolution are both plot organized. The work of Thorndyke (1977) has been validated and recognized in the field of cognitive science and controls for occurrences of instrumentation unreliability. The work of Seifert, Dyer, and Black (1986) identified story feature they called a thematic abstract unit (TAU). Thematic abstract units are 
adage-like statements represent abstract narrative comprehension (Seifert, Dyer, \& Black, 1986) while theme is plot organized and represents concrete narrative understanding (Thorndyke, 1977).

The work of Seifert, Dyer, and Black (1986) has been validated and recognized in the field of artificial intelligence. The measurement of story features was coordinated by this researcher and supported by an expert in the field of reading. A complete discussion of the story feature measurement follows in the Scoring Procedures section of this dissertation.

The transitivity measurement was derived from the work of Hopper and Thompson $(1980,1984)$ although they do not assign actual values to transitivity features. The transitivity scale is a continuum with a true zero point. Therefore, the transitivity measure represents a ratio scale. A ratio scale is the most precise level of measurement and allows for the use of inferential statistics as a of data analysis. The transitivity scale measurement includes the following:

\section{ACTIVE SENTENCE CONSTRUCTION}

\section{VERB}

dynamic verb $3 \mathrm{pts}$

sensory verb 2 pts

mental state 2 pts

other stative 2 pts

copula $\quad 1$ pts

\section{SUBJECT}

concrete, definite $\quad 3$ pts

generic, concrete 2 pts

abstract $1 \mathrm{pt}$

\section{OBJECT}

affected direct object 2 pts 
unaffected direct object $\quad 1 \mathrm{pt}$

subject complement $\quad 0 \mathrm{pt}$

\section{PASSIVE SENTENCE CONSTRUCTION}

\section{VERB}

dynamic verb 3 pts

sensory verb $2 \mathrm{pts}$

stative verb $2 \mathrm{pts}$

\section{SUBJECT}

$\begin{array}{ll}\text { affected subject } & 2 \mathrm{pts} \\ \text { unaffected subject } \quad 1 \mathrm{pt} & \\ \text { AGENT } \backslash \text { INSTRUMENT } & \\ \text { agent concrete, definite } & 3 \mathrm{pts} \\ \text { agent generic } & 2 \mathrm{pts} \\ \text { agent abstract } & 1 \mathrm{pt} \\ \text { agentless } & 0 \mathrm{pt}\end{array}$

The transitivity scale measurement used for this study was developed by Dr. Lynn Berk and this researcher, subsequently modified and applied by this researcher. It controls for occurrences of instrumental unreliability by interrater consensus. Specific discussion about the development of this transitivity scale following in the Scoring Procedures section of this dissertation.

Manipulation of the independent variables, constrained summary and the unconstrained summary, is a major characteristic of an experimental design. An experiment is valid only if the results obtained are due only to the manipulated independent variable and if they are generalizable to situations outside of the experimental setting. In this experimental design there was a need to control for the influence of any variable other than the independent variable on the dependent variables of story structure features produced and transitivity 
syntactic features produced. Control factors for extraneous variables included: subject, randomization of summary and story order, and time. For this study subject variables and treatment group variables were controlled for by using subjects from four college freshmen English classes. In order to be placed in a freshman English class the subjects are generally considered to have basic reading and writing skills, need a S. A. T. score of 960 , and a high school grade average of at least 2.5. This helps to control for unwanted differences between the subjects and helps to ensure that the groups' reading and writing characteristics are as equal as possible on all variables except the dependent variables of story structure and transitivity. Time variables were controlled for because each experimental session occurred within a two week period and each experimental session lasted for one hour and twenty minutes. Further, a repeated measures experimental design was used for this study with each subject reading both stories and exposed to both levels of summary. Counterbalancing the order of texts and tasks also controls for any effect of story order or summary order on the observed effect of both summary methods.

Design

The design for this study is presented in Table 3 : 
Table 3

Design for Independent Variables Used in Data Analysis

\section{Order}

First

Second
Story
Summary
Story
Summary

Treatment

\begin{tabular}{lllll}
\hline 1 & P & C & F & UC \\
2 & P & UC & F & C \\
3 & F & UC & P & C \\
4 & F & C & P & UC \\
\hline
\end{tabular}

Note: $\mathrm{P}$ stands for "Mrs. Packletide's Tiger", $\underline{\mathrm{F}}$ stands for "Fate", UC stands for unconstrained summary and $\mathrm{C}$ stands for constrained summary.

In the Summary Order column each summary cell is identified as being a constrained summary or an unconstrained summary. If an unconstrained summary precedes a constrained summary it shows that the unconstrained summary was presented first to the subject and a constrained summary was presented second to the subject. If constrained summary precedes unconstrained summary it shows that constrained summary was presented first and unconstrained summary was presented second to the subject. In the Story Order column each story order cell is identified by the letter F or P. The P represents the story "Mrs. Packletide's Tiger" and F represents the story "Fate". If $\mathrm{P}$ precedes $\mathrm{F}$ it shows that story $\mathrm{P}$ was presented first to the subject and story $F$ was presented second to the subject. If $F$ precedes $P$ it shows that story $\mathrm{F}$ was presented first to the subject and story $\mathrm{P}$ was presented second to the subject. The numbers $1,2,3$, and 4 represent the presentation of summary order and story order. For 
instance cell 1 indicates that story $\mathrm{P}$ was presented first and followed by a constrained summary and story $\mathrm{F}$ was presented second and followed by an unconstrained summary. Cell 2 indicates that story $\mathrm{P}$ was presented first and followed by an unconstrained summary and story $\mathrm{P}$ was presented second followed by a constrained summary. Cell 3 indicates that story F was presented first followed by an unconstrained summary and story $\mathrm{P}$ was presented second followed by a constrained summary. Cell 4 indicates that story $\mathrm{F}$ was presented first followed by a constrained summary and story $\mathrm{P}$ was presented second followed by an unconstrained summary. Story order and summary order had to be counterbalanced to account for any practice effect, fatigue, and learning.

Thus, each subject read both texts and wrote a constrained summary for one text and an unconstrained summary for the other text producing a repeated measures experimental design. This design was selected over a single-measure design for the following reasons: (a) to control for any possible treatment group variable effects, (b) to generalize beyond single text use, and (c) to examine within subject .

The features of internal validity include: (a) history, (b) maturation, (c) testing, and (d) instrumentation. Threats to internal validity have been controlled for in this study in the following ways:

1. Threats of history or maturation effects of this experiment were controlled by conducting the research within a two week period in February.

2. To prevent any test results due from pretesting effects, there was a preliminary short practice in summary writing done at the same time as the treatments. The short story, "The King and the Shirt" by Leo Tolstory was read aloud to the subjects. A copy of this story can be found in Appendix B.

Subjects

Seventy-two college freshmen participated in this experiment. All of the subjects were enrolled in college freshmen English classes at Florida International University, Miami, 
Florida. The subjects were members of four different college freshmen English classes. Admission criteria for Freshmen include minimum score of 950 on the SAT and a high school average of at least 2.5. The following information in Table 4 was gathered from the subjects in reference to their native language and if their native language was not English, what their native language was and when they learned to speak English. 
Table 4

Age English Was Learned and Subject Native Language by Age

Age that subject learned English?

Native language of subject

Age \# \# Language

$\begin{array}{llll}0-2 & 1 & 1 & \text { Arabic } \\ 2-4 & 3 & 3 & \text { Spanish } \\ 4-6 & 12 & 11 & \text { Spanish } \\ & & 1 & \text { Portuguese } \\ 6-8 & 3 & 3 & \text { Spanish } \\ 8-10 & 6 & 5 & \text { Spanish } \\ 10-12 & 4 & 1 & \text { Greek } \\ & & 2 & \text { Spanish } \\ & & 1 & \text { French } \\ 12-14 & 10 & 1 & \text { Portuguese } \\ & & 9 & \text { Spanish } \\ 15+ & 4 & 1 & \text { Chinese } \\ \text { Total } & 43 & 4 & \text { Spanish }\end{array}$

Note. Twenty-nine of the students were native English speakers while 43 were not native English speakers. Of these 43, 29 learned to speak English before the age of puberty. Materials

Materials were randomized and counterbalanced ahead of time using, the following procedures. Each general directions cover sheet, each story ( $\mathrm{P}$ and $\mathrm{F}$ ), each summary type 
(short and long), each biographical information sheet, metacognitive questionnaire and each closing written directions were placed in eight piles.

The first treatment group was formed as follows: cover sheet, story p, one sentence summary form, biographical information sheet, story $f$, unconstrained summary form, written metacognitive questionnaire, and closing written directions.

The second treatment group was formed as follows: cover sheet, story $p$, unconstrained summary form, biographical information sheet, story $f$, constrained summary form, written metacognitive questionnaire, and closing written directions.

The third treatment groups was formed as follows: cover sheet, story f, unconstrained summary form, biographical information sheet, story $p$, constrained summary form, written metacognitive questionnaire and closing written directions.

The fourth treatment group was formed as follows: cover sheet, story $f$, constrained summary form, biographical information sheet, story $p$, unconstrained summary form, written metacognitive questionnaire and closing written directions.

The treatment-group-ordered packets $(1,2,3,4,1,2$, etc.) were passed out to the subjects in the freshman English classes after their introduction to the experimental session and a short practice session. When the experiment was carried out at the next class the researcher simply continued passing out the next treatment group packet. This procedure continued for all of the subjects in each of the four participating college freshman English classes. Because of this randomization procedure all subjects were evenly distributed over the four treatment groups.

The materials used for this experiment consisted of a fifteen page packet for the four treatment groups. The short stories used for this experiment were "Mrs. Packletide's Tiger" (story p) and "Fate" (story f). Both of these short stories were written by Sake. It was determined by this researcher and an experts in the fields of reading and syntax that these stories embodied what was considered to be appropriate college freshmen literary material and 
both stories adhered to the constraints consistent with the elements of story structure such as setting, plot, resolution and theme. Both short stories contained themes that focus on planning failures, specifically both theme represent expectation failures which occur due to error in the protagonist's planning. For example, Mrs. Packletide never expected that her assistant would blackmail her, and Rex never expected to lose the important billiards game. Both of these stories provide the story structure and content features that could produce thematic abstract unit. Further, both of the stories used are naturally occurring text and represent appropriate college freshmen reading material.

The first page of each packet contained general direction for the experiment. These were identical regardless of the treatment group. The first to third page of treatment groups 1 and 2 contained story P. The first to fifth page of treatment groups 3 and 4 contained story F. The fourth page of treatment group 1 contained a constrained summary form and the fourth page of treatment group 2 contained an unconstrained summary form. The sixth page of treatment group 3 contained an unconstrained summary form and the sixth page of treatment group 4 contained a constrained summary form. The fifth page of treatment groups 1 and 2 contained a biographical information form containing spaces for information about the subjects native language. The biographical information form was used as a buffer task to extinguish possible initial summary form effects. The seventh page of treatment groups 3 and 4 contained a biographical information form. The seventh through eleventh pages of treatment groups 1 and 2 contained story $\mathrm{F}$ and the twelfth page contained for treatment group 1 an unconstrained summary form and treatment group 2 contained a constrained summary form. The eighth through tenth pages of treatment groups 3 and 4 contained story F. The eleventh page of treatment group 3 contained a constrained summary form and the twelfth page of treatment group 4 contained an unconstrained summary form. The thirteenth page of treatment groups $1,2,3$ and 4 contained a written metacognitive questionnaire form and the fifteenth page contained general closing directions. The purpose of the metacognitive 
questionnaire was to examine the subjects introspections about strategies used in the production of both summaries. The materials in each packet were designed for group administration regardless of treatment group.

Procedure

The time taken for the experiment in each of the four college freshman English classes was seventy-five minutes. The teacher in each class took approximately five minutes to take class roll. The researcher's introduction for this experiment and the practice session for this experiment took approximately fifteen minutes for each classroom, and the time to complete the experimental packets took approximately fifty-five minutes. Each of the four experimental sessions began with the previously presented general directions. All of the subjects in each of the four college freshman English classes received the same general introduction instructions and the same practice sessions.

The practice session procedure follows:

Hello, my name is Jane Devick, and I am conducting research for my doctoral dissertation in the field of education. I will be asking you to read two stories and write two different types of story summaries. First, you will all participate in a short summary writing practice session. When you have completed the practice session you will be given your story packets and asked to begin your reading. Are there any questions?

These directions were stated to provide a normal setting for this task. The task was placed into the general framework of a classroom setting to increase the generalizability of the results. None of the subjects in any of the four classroom had any questions. Once the general directions were read by the researcher the practice session began. These directions were stated:

You will be writing two types of story summaries: a one sentence summary and a free retelling summary. A one sentence summary consists of one sentence 
between five and fifteen words which includes what you consider to be important about the whole story. A free retelling summary consists of multiple sentences containing at least three sentences which includes what you consider to be important about the whole story. There are no right or wrong responses with either of these summaries. Are there any questions?

These directions were given to all subjects and were read by the researcher. None of the subjects had any questions. These directions simply made the difference between both types of summaries a matter of differences in the number of sentences. After the general practice session directions were given the researcher read, "The King and the Shirt" by Leo Tolstoy to the subjects. This is a very brief story that has no content similarities with either Sake stories used in this study. The following was stated to the subjects:

Now, let's listen to this story and afterwards we'll practice writing both types of summaries.

The story was read by the researcher to the subjects. These directions were given to make the instructional goal of this practice session clear to each of the subjects. After reading the practice story the following was stated to the subjects:

I will now distribute a sheet of paper to each of you for this practice session. First, let's write a constrained summary. A one sentence summary consists of one sentence between five and fifteen words and is what you consider to be important about the story. Are there any questions? Write a one sentence summary about the whole story I have just read to you.

At this point the subjects wrote a constrained summary. After they had written the constrained summary the following was stated to the subjects:

Ask yourself the following questions? Is this only one sentence? Is this one sentence comprised of one five to fifteen words? If you sentence has more than fifteen words rewrite the summary using only five to fifteen words. Go ahead do it now (pause). 
Ask yourself is this what I consider to be important about the story? If the answer is yes we are read to go on. Do you have any questions?

For this portion of the experiment only one subject in all of the four treatment groups commented. A subject in the fourth classroom began asking how she could shorten her sentence if it exceeded fifteen words. The researcher told her to shorten it to the best of her ability. The researcher made note of her packet when it was collected the packets. When I evaluated her packet she apparently did shorten her constrained summary to the appropriate length. Following this portion of the practice session the following was stated:

Now, let's write a free retelling summary of this story. Remember, a free-retelling summary consists of multiple sentences containing at least three sentences of what you consider to be important about the story. Are there any questions? Write a free retelling summary about the whole story I have just read to you.

When the subjects completed this portion of the practice session the following was stated:

Ask yourself the following questions. Did I use multiple sentences to write this summary? Did I use at least three sentences? If your free retelling summary does not contain at least three sentences rewrite the summary. Go ahead do it now (pause). Ask yourself is this what I consider to be important about this story? If the answer is yes we are ready to go one. Do you have any questions? Now, I will collect these papers and distribute to you your story packets. When you receive your packet you may begin. If you have any questions please come up to my desk. Thank-you. After the practice session the experimental packets were given to the subjects. The experimental packet portion for all four classes took fifty-five minutes. When the class session was over all of the packets were collected. A sample packet is provided in Appendix C. 


\section{Scoring Procedure}

When scoring the summary forms for the eighty-eight subjects initially in the experiment 16 subjects' summary forms had to be deleted. In treatment group I three subjects were deleted. One subject did not complete the packet and two subjects did not follow packet instructions. In treatment group II three subjects were deleted. Two subjects did not complete the packet and one subject did not follow packet instructions. In treatment group III six subjects were deleted. One subject included an inappropriate summary response, one subject had to leave during the experiment due to illness, two subjects failed to complete the packet, and two subjects did not follow packet instructions. In treatment group IV four subjects were deleted. Three subjects did not follow packet instructions and one subject did not complete the packet. All of the subjects who did not follow the packet and preliminary practice session directions failed to adhere to the length constraints of the unconstrained summary. It was stated during the practice session of this experiment that a unconstrained summary must contain at least three sentences. The deleted subjects unconstrained summaries included only one or two sentences.

The remaining seventy-two constrained and unconstrained summaries for each story were analyzed. The constrained summaries and unconstrained summaries for each story were analyzed for story structure features and transitivity features by two independent scorers. The story structure features contained three elements and the transitivity syntactic features contained eighteen elements subjected to a four-point scale.

\section{Story Scoring Rules}

For each item in the story structure features that were recalled, a score of one (1) was entered. Each item of story structure was only scored as being present and was scored as a one even if it was present several times during the recall. This was done to account for the length differences between a constrained summary and an unconstrained summary. For each item in the story structure elements that was not recalled, a score of zero was entered. The 
story features include plot, theme and thematic abstract unit. The story structure elements scoring rules are listed below:

1. The story structure elements discussed in Chapter I were identified in each summary form. For example:

Plot: A man who was always very poor in his upbringing (setting), He started a fire in this companions room (event).

Theme: She paid a high price to the villagers for the lion but the price of covering her life is indeed high.

Thematic Affect Unit: Fate is in our advantage...sometimes.

2. Each sentence was read and scored. However, occasionally each clause had to be scored separately when each clause contained a separate element of story structure. For example:

The importance of a woman killing a beast, (Plot) and the social recognition that came with it (TAU).

3. Every element of story structure was scored only once. If the element was present it was marked with a one and if the story structure element was not present it was marked with a zero. For detailed examples of the story structure features scoring procedures see Appendix D.

\section{Transitivity Scoring Rules}

The transitivity scale syntactic features as discussed in Chapter I were identified in each summary form. The following transitivity scale measurement was developed by Dr. Lynn Berk, a syntactician and this researcher and was modified by this researcher. Through examination of pilot study samples of constrained and unconstrained summaries, the transitivity scale was modified and implemented by this researcher and an expert in the field of reading. The transitivity scale instrumentation was derived from the works of Hopper and Thompson (1980; 1984). This scale is much simpler than the Hopper and Thompson (1980, 
1984) scale as it focuses exclusively on the quality of verb, subject, and object. Thus, it combines a number of features that Hopper and Thompson $(1980,1984)$ treat separately. The transitivity syntactic features were scalar. The items contained in the transitivity scale were either scored as a one (1), two (2), or three (3). A transitivity score was obtained for each summary form. The transitivity scale score for each summary was added and divided by the total number of clauses. Thus, the final transitivity score was obtained. This was done to account for the length differences between a constrained summary and an unconstrained summary. The following is the set of transitivity scoring rules:

1. Each summary was divided into clauses. A clause was defined as existing only if a verb was used. Thus, a sentence with two agents such as: Rex and the man played pool, was considered to have one clause while the following sentence had two clauses: Rex set the woman on the table and stopped the game.

2. Each clause was scored to determine what transitivity scale syntactic features were present. Clause refers to a group of words containing a verb.

3. Each feature of the transitivity scale received a specific score as follows:

\section{ACTIVE SENTENCE CONSTRUCTION}

VERB

$\begin{array}{ll}\text { dynamic verb } & 3 \text { pts } \\ \text { sensory verb } & 2 \mathrm{pts} \\ \text { mental state } & 2 \mathrm{pts} \\ \text { other stative } & 2 \mathrm{pts} \\ \text { copula } & 1 \mathrm{pts}\end{array}$

Application of the notion of highly transitive features asserts that a highly action/event embodied in a verb is characterized by a concrete action that is volitionally transferred from the subject to a totally affected object and results in a transitivity value of 3 . In that context sensory verbs, mental state verbs, and stative verbs clearly are less transitive than dynamic 
verbs resulting in a lower transitivity score value of 2 . Further, as transitivity is referred to as the transferred action from a subject to an object, a copula verb does not require an object but requires a subject complement and thus is detransitized resulting in a lower transitivity score value of 1 .

\section{SUBJECT}

$\begin{array}{ll}\text { concrete, definite } & 3 \mathrm{pts} \\ \text { generic, concrete } & 2 \mathrm{pts} \\ \text { abstract } & 1 \mathrm{pt}\end{array}$

A concrete, definite subject incorporates the following Hopper and Thompson (1980; 1984) transitivity features: (a) participant, (b) volitional,(c) agency and (d) individuation resulting in a high transitivity score value of 3 . A generic, concrete subject incorporates the following Hopper and Thompson $(1980,1984)$ transitivity feature of a participant resulting in a lower transitivity score value of 2 . Abstract subjects only incorporates from the Hopper and Thompson transitivity features that the subject syntactic slot is filled with a nonparticipant, nonvolitional, nonagentive, and o-individuation resulting in a lower transitivity score value of 1.
OBJECT
affected direct object
2 pts
unaffected direct object $\quad 1 \mathrm{pt}$
subject complement
$0 \mathrm{pt}$

An affected direct object incorporates the following Hopper and Thompson transitivity features: (a) participant, (b) individuation, and (c) affectedness resulting in a high transitivity score value of 2. An unaffected direct object incorporates the following Hopper and Thompson transitivity features: (a) participant and (b) individuation resulting in a lower transitive score value of 1 . A subject complement as it is not an object does not incorporates any of Hopper and Thompson's transitivity features resulting in a transitivity score value of 0 . 


\section{PASSIVE SENTENCE CONSTRUCTION}

VERB

dynamic verb $3 p t s$

sensory verb 2 pts

stative verb 2 pts

SUBJECT

affected subject $\quad 2 \mathrm{pts}$

unaffected subject $1 \mathrm{pt}$

AGENT $\backslash$ INSTRUMENT

agent concrete, definite 3 pts

agent generic 2 pts

agent abstract $1 \mathrm{pt}$

agentless $\quad 0 \mathrm{pt}$

4. For each summary form each time an element of the transitivity scale was present it received a score of one(1), two (2), or three(3) accordingly.

5. For each summary a total transitivity score was obtained. The total transitivity score was obtained by adding all of the scores together for each summary.

6. The total transitivity scale score was divided by the total number of clauses in each summary form. In this way a final transitivity/clause scale was obtained for each summary form, that was unaffected by the differences in the length of the two summary types. For detailed examples of the transitivity feature scoring procedures see Appendix E.

The recalls of the 72 subjects were scored by the researcher and an expert in the field of reading. The scorers worked separately and then as a team. Each team member initially scored the summary alone, and next the summaries were scored together to eliminate error in scoring. The scorers made the two following changes to the original transitivity scale: (a) the minus 1 points for a modality marker and negation were removed because it was considered 
not be relevant to transitivity features encountered in the data and (b) examination of relative clauses were removed because such clauses were considered to be irrelevant in the examination of the data. Conflicting scores were discussed and through a conference a final score was obtained. Even when modality, negation, and relative clauses were not considered, constrained summaries are statistically lower in transitivity than unconstrained summaries. Therefore, it was not necessary to complicate analysis by considering modality, negation, and relative clauses in scoring. A scoring consensus was reached by both of the scores through item by item discussion. Both scorers agreed to collapse setting and resolution into the plot category for conducting the statistical procedures for the following reasons: (a) according to Thorndyke (1977) setting and resolution are organization features of plot, (b) the purpose of this study was to examine summary differences between plot and thematic features, and (c) qualitative analysis would be carried out to examine the separate story features of setting, resolution, plot, theme and thematic abstract unit.

The raw scores thus computed were subjected to statistical analysis. The story structure elements were tested for significant differences due to treatment using a chi-square procedure since the data were nominal. The mean transitivity per clause scores were tested for significance using a $t$ - test. The results of these analysis are presented in the next chapter. 
Chapter IV

Results

Analysis and discussion of the results of the effects of the independent variables (constrained summary and unconstrained summary) upon the dependent variables (story structure features of plot, theme, thematic abstract unit and transitivity features) are reported in this chapter. The results are reported in two phases. The first phase of this study investigated the effects of story order and summary order on the subjects' constrained summary and unconstrained summary patterns to eliminate possible differences due to variables not studied but part of methodology.. Story Order by Summary Order effects are referred to as Treatments for the remainder of this discussion. A detailed examination of the Phase I follows. The second phase of results, reports the effects of the independent variables (constrained summary and unconstrained summary) on the dependent variables (story structure elements and transitivity features) without regard to order.

\section{Phase I}

Initially for Phase I of the results, treatments were analyzed according to the design shown in Table 5. 
Table 5

Design for Independent Variables Used in Data Analysis

Presented First

Presented Second

Story

Summary

Story

Summary

Treatment

\begin{tabular}{lllll}
\hline 1 & $\mathrm{P}$ & $\mathrm{C}$ & $\mathrm{F}$ & $\mathrm{UC}$ \\
2 & $\mathrm{P}$ & $\mathrm{UC}$ & $\mathrm{F}$ & $\mathrm{C}$ \\
3 & $\mathrm{~F}$ & $\mathrm{UC}$ & $\mathrm{P}$ & $\mathrm{C}$ \\
4 & $\mathrm{~F}$ & $\mathrm{C}$ & $\mathrm{P}$ & $\mathrm{UC}$ \\
\hline
\end{tabular}

Note. $\mathrm{P}$ stands for "Mrs. Packletide's Tiger", E stands for "Fate", UC stands for unconstrained summary and $\underline{\mathrm{C}}$ stands for constrained summary.

It was hoped that there would not be statistically significant differences in summaries due to the order in which the stories were read or due to the order in which the summaries were written. If this were shown, the data could be collapsed and the differences noted in the two types of summaries could be considered as order and text independent .

The initial investigation of treatment effects on story structure elements were conducted by chi-square tests to compare the proportions of usage in the four treatments. The first chi square test investigated the effect of the four treatments upon use of the constrained summary thematic abstract unit (STAU). The results of this test are shown in Appendix F Table 1. The results were not significant, $X^{2}(3, \underline{N}=72)=0.64, \mathfrak{p}<.89$. These results 
indicated that there were no treatment effects for use on the content of the constrained summary. Overall $65 \%$ used constrained thematic abstract units (STAU).

The next chi-square test investigated the effect of the four treatments upon the use of constrained summary plot feature (SP). The results of this comparison are shown in Appendix F Table 2. The results were not significant, $\mathbf{X}^{2}(3, \mathrm{~N}=72)=0.97, \mathfrak{p}<.81$, indicating no treatment effect for short plot features. Overall $33.3 \%$ of the treatment groups used constrained plot (SP).

The next chi-square test investigated the effect of the four treatments upon usage of the constrained summary theme features (ST). The chi-square test was not valid due to the fact that only two cases $(2.8 \%)$ of usage of ST were reported. The results of this comparison are shown in Appendix F Table 3.

The next chi-square test investigated the effect of the four treatments upon usage of the constrained summary default category, other (SO). The chi-square test was not valid due to the fact that only two cases $(2.8 \%)$ of usage of SO were reported. The results of this comparison are shown in Appendix F Table 4.

The next chi-square test investigated the effect of the four treatments upon the usage of the unconstrained summary thematic abstract units (LTAU). The chi-square test was not valid due to the small number using LTAU. Treatment 1 reported 2 cases, treatment 2 reported 3 cases, treatment 3 reported 4 cases and treatment 4 reported 4 cases. Overall $18.1 \%$ of the sample used unconstrained summary thematic abstract units (See Appendix F Table 5)

The next chi-square test investigated the effect of the four treatments upon the usage of unconstrained summary plot features (LP). The chi-square test was not valid due to the fact that only one case ( $1.4 \%$ ) did not use LP. Overall $98.6 \%$ of the sample used LP (See Appendix F Table 6). 
The next chi-square test investigated the effect of the four treatments upon the usage of unconstrained summary theme features (LT). The chi-square test was not valid due to the fact that only five (6.9\%) cases used unconstrained summary theme (See Appendix F Table 7). However, because the five cases were not distributed uniformly across the four treatments additional tests were carried out, grouping the treatments by story order and summary order.

Fisher's Exact Tests were carried out to investigate the effects of story order (treatments 1,2 versus treatments 3,4) and summary order (treatments 1,4 vs treatments 2,3). There was no significant effect for summary order upon unconstrained summary theme, $p<.46$. However, Fisher's Exact Test conducted for story order by usage of unconstrained summary theme was significant, $p<.02$ (See Appendix F Table 8 ). Five $(14.7 \%)$ used unconstrained summary theme for Fate/ Mrs. Packletide's but none did for Mrs. Packletide's Tiger/Fate. These results may be due to the different number of episodes included in both stories and which episode was considered to be more memorable for the subjects. A complete discussion is carried out in the Qualitative Results Chapter.

The next chi-square test investigated the effect of the four treatments upon usage of the unconstrained summary default category, other (LO). The chi-square test was not valid due to the fact that only four cases $(5.6 \%)$ of LO were reported. The results of this comparison are shown in Appendix F Table 9.

The next investigation of treatment effects were on the number of transitivity features (ST) for constrained and unconstrained summaries conducted by an analysis of variance. Results of the analysis on the number of constrained summary transitivity features by treatments were not significant, $\mathrm{E}(3,68)=0.19, \mathrm{p}<.91$ (See Appendix F Table 10) and analysis of results for unconstrained summary transitivity features by treatments were not significant, $\mathrm{F}(3,68)=0.60, \mathrm{p}<.615$ (see Appendix F Table 11). 


\section{Phase II}

The results for Phase I indicated that there were no significant effects for story order and summary order on the dependent variables except in the case of using unconstrained summary theme and story order. Thus, Phase II of the statistical examination of the effect of the independent variables on the dependent variables was conducted. The data was then collapsed into the two summary types independent of story and summary type order.

Phase II examined the effect of the independent variable (constrained summary or unconstrained summary) on the dependent variables of story structure elements and transitivity features. The differences found were due to differences in summary type. A Paired t-test was used to compare the difference between the mean numbers of constrained summary transitivity features (STC) used and the unconstrained summary transitivity features (LTC). The transitivity scale used for the scoring of the transitivity features is ratio scaled so therefore a t-test was appropriate to determine whether the two means were significantly different at a 5\% level. The results of the Paired t-test are below in Table 6:

Table 6

Transitivity Feature Means by Summary Type and Standard Deviations

Variable

Cases

Mean

SD

Unconstrained

Summary

72

5.17

0.56

Constrained

Summary

72

4.17

1.45

$* * * p<.001$ 
A significant difference was found between the constrained summary transitivity mean score (STC) and the unconstrained summary transitivity mean score (LTC), $\mathrm{t}(71)=5.72$, ${ }^{* * *} \mathrm{p}=<.001$. The higher mean (5.17) for the unconstrained summary indicates that more transitivity features were used per clause than in the constrained summary (4.17). Therefore, we reject the null hypothesis of no difference between the mean numbers of transitivity features used in a constrained summary and an unconstrained summary.

The standard deviation was used as an index of variability in the number of transitivity features used per clause. The variability in the unconstrained summaries was low, .56 with a range of 0 to 3 . This showed that the usage of transitivity features were consistent across subjects. However, the transitivity features used across constrained summaries were more variable with a standard deviation of 1.45 on the four point scale. This showed that the use of transitivity features varied within the constrained summary group. However, this variation did not effect the significance of low transitivity features produced in the constrained summaries.

In addition, ranges of transitivity scores between the constrained summary transitivity features (STC) and the unconstrained summary transitivity features were examined. The constrained summary (STC) transitivity features score had a minimum transitivity value of 1.5 while the minimum transitivity value for the unconstrained summary (LTC) had a minimum value of 3.5. Thirty-five percent of the STC scores fell below the lowest transitivity value of 3.5 for unconstrained summary (LTC) which indicated that the constrained summary (STC) produced many transitivity scores well below the minimum transitivity score for the unconstrained summary . The constrained summary transitivity features scores had a maximum transitivity value of 8.0 while the transitivity value for the unconstrained summaries had a maximum transitivity value of 6.5 . This showed that constrained summaries had an overall lower transitivity value than unconstrained summary. However, constrained summaries had a higher maximum transitivity value than unconstrained summaries. This is discussed in detail in Chapter 5. 
The next group of tests of significance examined the usage of the story structure elements of plot, thematic affect unit, theme, and the default category, other. This group of variables were tested using the nonparametric McNemar's Test. McNemar's test is used to compare correlated proportions. In this study these tests were used to examine the different proportions of subjects using story structure features in a constrained summary and in a unconstrained summary. The first analysis involved examining differences between percents and proportion of usage of constrained summary thematic abstract units (STAU) and the unconstrained summary thematic abstract units (LTAU). Table 7 shows the results:

Table 7

Percents and Proportion of Usage of Thematic Abstract Units in Summaries

\begin{tabular}{|c|c|c|c|c|c|c|}
\hline \multirow[t]{2}{*}{ Summary Type } & \multicolumn{2}{|c|}{ Not Used } & \multicolumn{2}{|c|}{ Used } & \multicolumn{2}{|c|}{ Total } \\
\hline & $\#$ & $\%$ & $\#$ & $\%$ & $\#$ & $\%$ \\
\hline Constrained & 25 & 34.7 & 47 & 65.3 & 72 & 100 \\
\hline Unconstrained & 59 & 81.9 & 13 & 18.1 & 72 & 100 \\
\hline
\end{tabular}

The test was significant, $X^{2}(1, \underline{N}=72)=30.25, * * * p<.001$, indicating a difference in proportions of subjects who used thematic abstract units (TAU) in the unconstrained summary and the constrained summary. Thus, the null hypotheses is rejected and the research hypothesis is supported. Examination of data shows that in the unconstrained summary only $18.1 \%$ of the subjects used thematic abstract units (LTAU) as compared to $65.3 \%$ in the constrained summaries. 
The next McNemar's test examined the differences between percents and proportion usage of plot features by summary type. Table 8 indicates the results:

Table 8

Percents and Proportion of Usage of Plot in Summaries

\begin{tabular}{|c|c|c|c|}
\hline \multirow[t]{2}{*}{ Summary Type } & Not Used & Used & Total \\
\hline & $\%$ & $\%$ & $\#$ \\
\hline
\end{tabular}

Constrained

$48 \quad 66.7$

$24 \quad 33.3$

$72 \quad 100$

$\begin{array}{lllllll}\text { Unconstrained } & 1 & 1.4 & 71 & 98.6 & 72 & 100\end{array}$

$* * * \mathrm{p}<.001$

The test was significant $X^{2}(1, N=72)=45.02, * * * p<.001$, indicating a difference in the proportion of subjects who used plot in the unconstrained summaries and the constrained summaries. Thus, the null hypothesis is rejected and the research hypothesis is supported. Examination of the data shows that in the unconstrained summary $98.6 \%$ of the subjects used plot features as compared to only $33.3 \%$ of the subjects in the constrained summary .

The next McNemar's test examined differences between theme usage by summary type. Table 9 indicates the results: 
Table 9

Percents and Proportion of Usage of Theme in Summaries

\begin{tabular}{lcccccc}
\hline Summary Type & \multicolumn{2}{c}{ Not Used } & \multicolumn{2}{c}{ Used } & \multicolumn{2}{c}{ Total } \\
& $\#$ & $\%$ & $\#$ & $\%$ & $\#$ & $\%$ \\
\hline Constrained & 70 & 97.2 & 2 & 2.8 & 72 & 100 \\
\hline Unconstrained & 67 & 93,1 & 5 & 6.9 & 72 & 100 \\
\hline
\end{tabular}

Note. Binomial test $p<.37$

The test was not significant $p<.37$, indicating that there was not a difference in the proportion of subjects who used theme in the unconstrained summaries and the constrained summary. Thus, the null hypothesis was not rejected. However, theme as a separate category was not of significance to this study as theme was considered to be an element of plot organization. According to Thorndyke (1977) theme is plot organized and is an element of plot organization.

The final McNemar's test examined differences in usage of "other" between the constrained summary and unconstrained summary. Table 10 indicates the results: 
Table 10

Percents and Proportion of Usage of Other in Summaries

\begin{tabular}{|c|c|c|c|c|c|c|}
\hline \multirow[t]{2}{*}{ Summary Type } & \multicolumn{2}{|c|}{ Not Used } & \multicolumn{2}{|c|}{ Used } & \multicolumn{2}{|c|}{ Total } \\
\hline & $\#$ & $\%$ & \# & $\%$ & $\#$ & $\%$ \\
\hline Constrained & 70 & 97.2 & 2 & 2.8 & 72 & 100 \\
\hline Unconstrained & 68 & 94.4 & 4 & 5.6 & 72 & 100 \\
\hline
\end{tabular}

Note. Binomial test $p<.625$

The test was not significant $p<.625$, indicating no difference in proportions of subjects who used the default category, other, in the unconstrained summary and the constrained summary.

For the purposes of comparison, percents of usage of thematic abstract units and plot for the constrained summary and unconstrained summary are presented respectively, in Table 11

Table 11

Percentages of Usage of Thematic Abstract Units and Plot by Summary Type

Unconstrained Summary

$\%$

Thematic Abstract Unit

Plot
$18.1 \%$

$98.6 \%$
Constrained Summary

$\%$ 
The results indicate support for several of the research hypotheses. It was found that: (1) the constrained summary yielded greater percentage of usage of thematic abstract units $(65.3 \%)$ as compared to the unconstrained summaries thematic abstract units (18.1\%), and (2) the unconstrained summaries contained a greater percentage of plot elements $(98.6 \%)$ as compared to the constrained summary plot elements (33.3\%).

Conclusions

The analysis conducted on the data from this study found that there was a significant transitivity feature mean difference between a constrained summary and an unconstrained summary, $\mathrm{t}(72)=5.72, * * * \underline{p}=<.001$, indicating that the number of transitivity features produced from each summary are task dependent. The constrained summaries contained fewer number of transitivity features while the unconstrained summaries contained a greater number of transitivity features on the averages. Further, the analysis found that there were differences between usage of thematic abstract units in a constrained summary and a unconstrained summary, $X^{2}(1, \underline{N}=72)=30.25, * * * p<.001$, indicating that use of thematic abstract units was task dependent. The constrained summaries produced a significantly greater percentage of students using thematic abstract units $(65.3 \%)$ than did the unconstrained summaries, (18.1\%). Finally, the analysis found that there were proportional differences in usage between plot features in a constrained summary and a unconstrained summary $\left.\chi^{2}(1, \underline{N}=72)=45.02, * * * p=<.001\right)$, indicating that the production of plot features was task dependent. The unconstrained summaries produced a significantly greater percentage of students using plot features $(98.6 \%)$ than did the constrained summaries, $(33.3 \%)$.

Constrained summaries are characterized as being more abstract/generic in nature and as being less transitive. Unconstrained summaries are more plot centered and, consequently, are more transitive. These two summary types also differed qualitatively. These will be explored in the next chapter. 


\section{Chapter V}

\section{Qualitative Results}

The purpose of this qualitative research is to provide insights into the patterns in the quantitative data and thinking strategies used by the subjects in the production of the summaries. A statistical analysis of the data cannot present a complete picture of the strategies used by the subjects in the production of the summaries.

The collecting of qualitative data from this study included analyses of the subjects' summaries and metacognitive questionnaires for semantic and syntactic features not accounted for by statistical analyses. Analyses of the summaries and the metacognitive questionnaires of the subjects were conducted by this researcher. The raw data was qualitatively examined in regard to story features and transitivity features contained in both summary types. For examination of the metacognitive questionnaire the researcher coded each piece of data to investigate how the subjects described the strategies that were used in the production of each summary. Next, the subjects description of strategies was separated into general categories that indicated subjects who shared the same orientation about their thinking strategies that were used in the production of each summary.

Semantic Features of the Unconstrained Summaries

Examination of the semantic features of the unconstrained summaries indicated that the story features represented in the unconstrained summaries contained canonical story structure elements: setting, goal, plot, theme, and resolution. Table 12 shows a breakdown of the number of each type of semantic story feature present in all of the unconstrained summaries: 
Table 12

Number and Type of Semantic Story Feature Contained in the Unconstrained Summaries

Story Feature

Number

Thematic Abstract Unit

12

Plot

61

Setting

33

Goal

45

Resolution

31

Theme

6

Total

188

Since there were only 72 subjects, this data indicates that most subjects used more than one story feature type. These results show that unconstrained summaries produce mostly plot organized features of story comprehension followed by goal, setting, resolution, thematic abstract units, and theme. In sum, this analysis demonstrated that unconstrained summaries contained a greater number of story grammar features (176) than thematic abstract units (12) and the majority of the unconstrained summaries contained canonical story structure elements.

These results imply that due to the length of the unconstrained summary the reader selected specific semantic strategies that encoded produced plot written statements. This further confirms the finding that the content of the unconstrained summaries tended to be similar to the prototypical story structure described in story grammar research. This kind of summary would be the result of readers using deletion, generalization, and integration strategies. These data are consistent with Brown and Day's (1983) research. 
In fact, this tendency to produce prototypical summaries was the source of a difference in the summaries for "Fate" and "Mrs. Packletide's Tiger". "Fate" fit the prototype completely because it is a single-goal story. Rex played pool to make money. However, "Mrs. Packletide's Tiger" is a two-goal story. Mr. Packletide's desire to shoot a tiger to make a favorable impression on her peers, and Mrs. Mebbin's decision to blackmail Mrs. Packletide because she had not really killed the tiger. In "Fate", the climax of the story, the location of the high-impact information was at the end of the story, the resolution to the pool game. In "Mrs. Packletide's Tiger", however, the climax occurred at the end of the first episode, but the story continued with the second episode.

Additional analysis of the unconstrained summaries supported Thorndyke's (1977) notion that all of the canonical features of story structure are plot organized. Following are examples of the story feature elements produced in the unconstrained summaries and an explanation of how each feature is typical of plot organization.

1. The following are two examples of a setting story feature:

Rex is a material man.

Rex is a man who obtained his goals.

These statements are typical of plot because it includes background information about the character and the characters goal and are consistent with Thorndyke's (1977) definition of plot.

2. The following are two examples of a goal story feature:

Rex was looking for a sure bet.

Mrs. Packletide wanted to shoot a tiger.

These goal statements too reflect character action directed toward a goal and are consistent with Thorndyke's (1977) definition of plot.

3. The following are two examples of resolution story features:

Rex threw her on a game table to stop the game and fire. 
At the end she gets tired of being a big shot.

4. The following are two examples of plot story features:

Rex tried to run away from the situation

Mrs. Packletide killed two animals.

This data supports that the canonical features of story organization represent plot organized story structure

Examination of the unconstrained summaries written about story "Fate" revealed that thirty-five out of thirty-five subjects made some type of reference to the first single climax which represented features of plot organization. The following are some examples:

$\ldots$ he was looking for a sure bet.

One day he decided to make the greatest bet of his life.

These types of responses demonstrate that the plot organization of a one climax story such as, "Fate" was consistently recalled by a majority of the subjects. This suggests that readers considered plot organization features to be most memorable. Further, this suggests that readers use semantic strategies that promote the use of an aestethic stance (Many, 1991) and reader affect theory (Brewer \& Lichtenstein, 1982) indicating features of story that were considered to be most memorable.

"Mrs. Packletide's Tiger" is a story that represents a two climax story. Climax one is about Mrs. Packletide's desire to shoot a tiger in order to win social recognition. The second climax is about how Mrs. Packletide is blackmailed by her longtime companion Mrs. Mebbin. The unconstrained summary results indicated that the subjects primarily recalled the first climax of the story, "Mrs. Packletide's Tiger". Twenty-five out of thirty-seven subjects in their unconstrained summaries recalled climax one of "Mrs. Packletide's Tiger " while only 10 of the subjects recalled climax two of "Mrs. Packletide's Tiger". These results suggest that the concrete action/event plot organization of climax one that involved, "the shooting or killing of a tiger" was better recalled by the subjects than the "blackmailing" an individual 
that represented climax two of "Mrs. Packletide's Tiger". Highly concrete, humorous plot events stand out for both structural importance and strong affective appeal (Brewer \& Lichtenstein, 1982). These results support the notion that concrete/action plot organized story features are better recalled by subjects than less action events, such as in "blackmailing someone" as was found in "Mrs. Packletide's Tiger". These results, too, support structural affect theory (Brewer \& Lichtenstein, 1980) and use of aesthetic stance (Many, 1991) as semantically encoding highly memorable story events that reflect affect understanding making them easy to recall.

These results imply that due to the length of the unconstrained summary, the reader selected specific semantic strategies that encoded produced plot written statements. This further confirms the finding that the content of the unconstrained summaries tended to be similar to the prototypical story structure described in story grammar research. This kind of summary would be the result of readers using deletion, generalization, and integration strategies. These data are consistent with Brown and Day's (1983) research. Syntactic Features of Unconstrained Summaries

Further examination of the unconstrained summary transitivity features showed that of the 176 plot features represented, each was considered to contain high transitivity features. In contrast, the twelve unconstrained summaries that contained thematic abstract units each had low transitivity features. This further substantiates the highly transitive nature of plot features and the minimally transitive nature of thematic abstract units.

Semantic Features of the Constrained Summaries

Generally, the constrained summaries contained more thematic abstract units than plot features. Table 13 represents a breakdown of the number of each type of story feature present in the constrained summaries: 
Table 13

Number and Type of Semantic Story Features Contained in the Constrained Summaries

Story Feature

Number

Thematic Abstract Unit

Setting

Goal

Resolution

Plot Other Events

Theme

These results show that constrained summaries contained more thematic abstract units than plot features as compared to unconstrained summaries which contained more plot features than thematic abstract units. These results imply that, due to the brevity of the constrained summary, the readers selected specific semantic strategies that encoded produced abstract thematic written statements.

Syntactic Features of Constrained Summaries

Examination of the data revealed that 33 out of 47 constrained summaries contained thematic abstract units and low transitivity features. However, fourteen constrained summaries that contained thematic abstract units were scored high in transitivity features. Semantic examination of these highly transitive constrained summaries revealed that syntactically each summary included a dynamic verb that was used in the context of an abstract subject. For instance:

You win some you lose some. 
In this clause, win which is generally used as a dynamic verb is used to represent an abstract result of an action. This action verb (win) is transferred from a nonagentive abstract subject (you) to an unaffected generic direct object (some). This supports the idea that during the comprehension and summary writing process, readers have access to semantic strategies and syntactic strategies that encode into a written statement.

Examination of the constrained summaries for "Mrs. Packletide's Tiger" revealed that twenty-four subjects out of thirty-seven subjects recalled thematic abstract units. Constrained summaries for "Fate" revealed that twenty-three out of thirty-five subjects recalled thematic abstract units. These findings indicate that constrained summaries produce thematic abstract units and act as an abstract filter in narrative comprehension that is not effected by the number of plot features represented in a narrative. In contrast, unconstrained summaries produce plot story features and act as a concrete filter in narrative comprehension that is effected by the number of plot features represented in a narrative. Unconstrained summaries produce recall that represents concrete narrative comprehension while constrained summaries produce recall that represents abstract narrative comprehension regardless of the number of plot features and high transitivity story episodes.

\section{Metacognitive Questionnaire}

The metacognitive questionnaire was included as the final page of the research packet. The following questions were contained in the metacognitive questionnaire.

1. How did you choose the information that you included in your free retelling?

2 . How did you choose the information that you included in your one sentence summary?

3. What do you consider to be the differences between a free retelling and a one sentence summary?

4. Would you change any of the information in your free retelling?

5 . Would you change any of the information in your one sentence summary? 


\section{Responses to Question 1}

The predominant responses by the subjects to question 1, "How did you choose the information that you included in your free retelling?" indicated that in the production of a unconstrained summary subjects considered the information recalled to be an overview of the main points of the narrative. Subjects generally responded by expressing that the information included in their unconstrained summaries was what they "remembered; included "facts about the story"; was an "overview ,summary, or overall concepts of the story"; included "sentences that tell about the story"; contained the "main points or interesting main points of the story"; was a "general idea supported by facts" about the story; was "information related to theme" about the story; and was related to the "character, climax and circumstances" of the story: Table 14 contains the number of responses and question category type produced by the subjects in relation to question 1.

Table 14.

Subjects Question 1 Category Type and Number of Responses

Question Category Number of Responses

Overview of Story

Main Points of Story

Best Remembered Parts

Facts about the Story

General Idea of the Story

Information Related to Theme

Information Related to Character

Total 
Generally, the responses to question 1 of the metacognitive interview demonstrated that the reported strategies used by the subjects in the production of a unconstrained summary was an overview of the main points of the story with some emphasis upon the character, setting, plot features, and climax of the story. These reported subject introspection's relates directly to Kintsch and van Dijk's (1977) generalization and integration macrorules. The generalization and the integration macrorules refer to summary production that includes a development of the main portions of discourse by lumping multiple examples together and including implicit story information.

\section{Responses to Question 2}

The predominant responses to question 2 by the subjects, "How did you choose the information that you included in your one sentence summary?" indicated that in the production of a constrained summary subjects considered the information recalled to be a global thematic message of the story. Subjects generally responded by expressing that the information included in their constrained summaries was what they considered to be "the general idea" about the story; "the theme, message, main concept, moral, and central factor" of the story; the "condensing of ideas about the story"; "the elimination of details about the story"; "information about the last part of the story"; and "what the story means not what happened in the story". Table 15 contains the number of responses and question category type produced by the subjects in relation to question 2 . 
Table 15.

Subjects Question 2 Category Type and Number of Responses

Question Category

Number of Responses

Main Idea of Story

6

Message of Story

10

Moral of Story

15

Global Message

7

General Idea

8

Theme

26

Total

72

Generally, the responses to question 2 of the metacognitive questionnaire demonstrated that the strategies used by the subjects in the production of a constrained summary were a global and condensed thematic message about what was considered to be important about the story. These types of responses are supported by Kintsch and Van Dijk's (1977) construction macrorule. The construction macrorule is a topic sentence that represents global and condensed text meaning.

Responses to Question 3

The predominant responses to question 3 by the subjects, "What do you consider to be the differences between a free retelling and a one sentence summary?" indicated that the production differences between a constrained summary and a unconstrained summary were differences of length constraints, clarity of idea and theme message versus plot features. Table 16 contains the number of responses and question category type produced by the subjects in relation to question 3 
Table 16

Subjects Question 3 Category Type, Number of Responses, and Summary Type

Constrained Summary

Unconstrained Summary

\begin{tabular}{lcll}
\hline Shorter & 10 & Longer & 11 \\
Clarity of Ideas & 10 & Too Many Details & 9 \\
Theme & 6 & Plot & 16 \\
Getting to the Point & 10 & Non-clarity & 3 \\
More General & 18 & Describes the Situation & 18 \\
Most Important Idea & 10 & Vivid & 3 \\
Condense Ideas & 15 & Information is in Text & 9 \\
\hline
\end{tabular}

Note. Some subjects included more than one summary difference.

Generally, the responses to question 3 of the metacognitive questionnaire demonstrated that the subjects considered to the differences between both summaries were that a constrained summary was shorter and represented the theme moral or point of the story while the unconstrained summary was longer and represented the details of the story. There is consistency between question 3 and the responses to questions 1 and 2 .

\section{Responses to Questions 4}

The predominant responses to question 4 by the subjects, "Would you change any of the information in your free retelling?" indicated that 64 of the subjects responded no to this question and only eight of the subjects responded yes to this question. The response made by these subjects indicated that they would have liked to reread the story and at that point they might have wanted to add some information. 


\section{Responses to Question 5}

The predominant responses to question by the subjects, "Would you change any of the information in your one sentence summary?" indicated that 66 of the subjects responded no to the question and only 6 of the 72 subjects responded yes to this question. Further, subjects that responded "yes" to question 4 also responded "yes" to question 5. The responses by these six subjects included the following statements;" Maybe I did not understand the main point"; " Absolutely I'm sure I can go home and think of a more exact sentence"; "I would need to read the story again"; "I would fine tune it a little more"; "Yes but less than" a unconstrained summary; and "yes" the subject did not elaborate. Summary of Qualitative Findings

The overall usefulness of the data from the questionnaire supports the belief that the subjects had a clear understanding of the differences in the tasks of creating a constrained and an unconstrained summary. The qualitative examination of data reveal that generally the unconstrained summaries contained canonical story features and high transitivity features. Also, setting, goal, theme, and resolution are typical within plot organization. This is supported by the works of Thorndyke (1977). Also, unconstrained summary plot elements are encoded by the readers use of a semantic aesthetic stance and reader affect strategy. On the other hand, a constrained summary causes the reader to produce an abstract recall in the form of thematic abstract units and low transitivity features. This was supported by the number of thematic abstract units produced by a constrained summary as compared to an unconstrained summary. Another finding of this qualitative examination of the data collected supports that when a story has two climaxes one abstract in nature and the other concrete in nature as found in "Mrs. Packletide's Tiger" an unconstrained summary acts as a filter to produce concrete plot organized summaries while a constrained summary acts as a filter to produce the abstract thematic abstract summaries. 
The conclusions drawn from the qualitative examination of the metacognitive questionnaires indicate that readers do not seem to be consciously aware of how they make sense of text because so much of processing is at a sub-conscious level. The questionnaires accounted for reported subject introspected differences between both summary types such as length and topic distinctions rather than syntactic, memory and story structure distinctions. However, subjects consistently stated that an unconstrained summary was more of an overview of the main parts of the story while a constrained summary was the moral, message, or main concept of the story. 


\section{Chapter VI}

\section{General Discussion}

\section{The Questions Answered}

At this point there is a need to return to the original research questions asked at the onset of this study. The first research question was, "How do the story structure features of a constrained summary and a unconstrained summary differ? Is there a consistent pattern in these differences? The results of this research demonstrated that there were consistent story structure feature differences between summary types. The analysis of the data found that the constrained summaries produced a significantly greater percentage usage of thematic abstract units, $(65.3 \%)$ in contrast to unconstrained summaries, $(18.1 \%)$. The analysis on the data from this study found that constrained summaries produced only $33.3 \%$ usage of plot features in contrast to the unconstrained summaries that produced $98.6 \%$ of plot features. The story feature elements of both summaries differed and the null hypotheses were rejected. The research hypothesis that states that an unconstrained summary would contain predominantly plot features because of its length is supported as well as the research hypothesis that a constrained summary will contain predominantly thematic abstract units because of the constraints on its length is supported. Constrained summaries contained more thematic abstract units while unconstrained summaries contained more plot features

The second research question was, "How does the transitivity of a constrained summary and a unconstrained summary differ? Is there a consistent pattern in the differences? The results demonstrated that there were consistent transitivity value differences between both summary types. The analysis of the data from this study found that there was a difference between summaries $\mathrm{t}(71)=5.72, * * * \mathrm{p}<.001$. The differences indicated that the mean transitivity score for the unconstrained summary was one point above the mean for constrained summary and the constrained summary had a minimum transitivity value of 1.5 while the minimum transitivity value for the unconstrained summary was 3.5 . These results 
indicated that constrained summaries produced lower transitivity values on the average than unconstrained summaries and the null hypothesis was rejected. The research hypothesis that stated that a constrained summary would be written using low transitivity features was supported as well as the research hypothesis that an unconstrained summary will be written using highly transitive features thus reflecting action orientation of summary because of its length is supported. The results also indicated that unconstrained summaries had smaller variability $\mathrm{SD}=0.56$ than constrained summary $\mathrm{SD}=1.45$. This indicated that there was more of a consistent pattern for unconstrained summaries than constrained summaries. This was due to the use of dynamic verbs in abstract contexts and is further examined in Appendix G.

The third research question was, "What do the subjects report about their processing of a constrained summary and an unconstrained summary?". The results of the metacognitive questionnaire indicated that the subjects were not aware of the cognitive processes differences between both summaries. Subjects reported that they used a condensed global and theme generating strategy when producing a constrained summary. In contrast, when producing an unconstrained summary the metacognitive questionnaire results indicated that the subjects used a overview description of main points and plot generating strategy

The fourth research question was, "Are the differences in story features and syntactic features produced by both summaries task dependent? Story feature results demonstrated that subjects used more thematic abstract units in the production of a constrained summary, $(65.3 \%)$ than during the production of a unconstrained summary, $(18.1 \%)$. Subjects used more plot features in the production of a unconstrained summary, (98.6\%) than during the production of a constrained summary, (33.3\%).

The data suggests that readers are able to construct multiple meanings of narrative understanding. Readers can create understanding of narrative text through the simultaneous use of semantic strategies and syntactic features which are encoded into a written statement. 
Also, use of a particular narrative task acts as a filter from which readers subconsciously make either concrete or abstract, semantic and syntactic choices that encode into a written statement. Thus, comprehension processes and products are of equal status. Ultimately, it needs to be understood how readers have available to them multiple ways in which to construct narrative understanding.

Multidimensional Theory of Comprehension

The quantitative and qualitative data collected for this study has helped to validate the multidimensional theory of narrative comprehension. The theory proposes that readers comprehend narratives at many levels simultaneously. This study showed that readers use specific semantic choices and syntactic features simultaneously in the production of each summary type.

This theory asserts that during the summary production process readers have available to them multiple levels of meaning, a variety of semantic strategies, and many syntactic choices from which to construct meaning. Each summary task acts as a kind of filter to promote the reader's use of semantic strategies and syntax features that encodes into a written statement.

The semantic strategies used by readers during the production of a summary include elements of story content features, memory organizational packets (MOPs), thematic organizational points (TOPs), thematic abstract units (TAUs), affect units, reader stances (aesthetic and efferent) and macrorules (integration, generalization, and construction). It is suggested that the reader's use of a specific macrorule strategy promotes selection of syntax strategies.

Syntax strategies used by readers during the production of a summary include transitivity features (high and low) as well as other story syntax features such as cohesion (e.g. tense, sequence, markers, and signaling of foregrounded information). In producing a summary, the reader selects semantic information that is associated with syntactic information. 
Thus, the differences in syntactic information such as transitivity features and story structure features emerge. The task selected, constrained summary or unconstrained summary produced differences that were dependent upon the specific semantic information that activates specific syntactic information. The semantic and syntax strategies used by readers is shown in Figure 3.

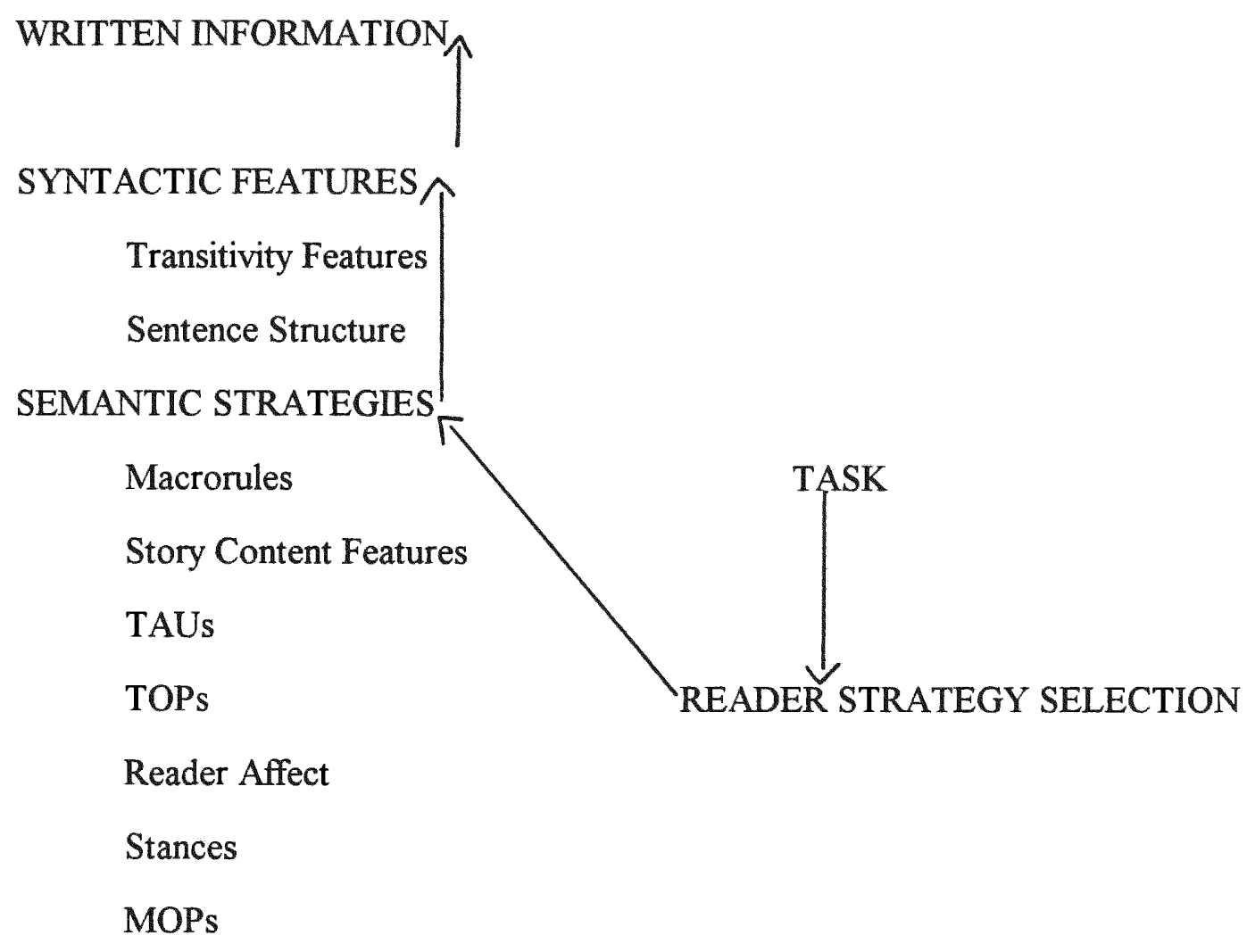

Figure 3 . Semantic and syntax strategies used by readers during the production of a summary task.

According to Figure 3 the older reader uses semantic strategies and syntax strategies during the production of narrative summaries. According to Figure 3 during the production of a narrative constrained summary the older reader uses specific abstract semantic strategies such as, story content features, thematic organizational points (TOPs), thematic abstract units 
(TAUs), efferent stance, and construction macrorule which promotes the reader's use of syntax strategies such as, low transitivity features, story structure features such as cohesion. During the production of an unconstrained summary the older reader uses specific concrete strategies such as story content features, memory organizational packets (MOPs) the aesthetic stance, reader affect responses, and the integration and construction macrorule which promotes the reader's use of syntax strategies such as, high transitivity, and sentence structure. Thus, an unconstrained summary due to its length promotes specific coordination of information while simultaneously producing concrete story comprehension represented by plot features and high transitivity features while a constrained summary due to brevity promotes coordination of information while simultaneously producing abstract story comprehension represented by thematic abstract units and low transitivity features.

The comprehension of narratives is a complicated process. This research begins to examine the multidimensional aspects of narrative comprehension. The multidimensional theory of narrative comprehension has been supported by the empirical and theoretical findings of this research. This theory only begins to postulate how a concept of narration is stored in the readers memory and is cognitively constructed by the reader. The general findings of this research emphasize the importance of narrative comprehension tasks that activate different models of narrative understanding. One must take into account the different semantic and syntactic strategies related to narrative summary comprehension. The following is a discussion of the theory that underlies narrative summary comprehension semantic strategies and syntactic features used by readers.

\section{Semantic Story Understanding}

The work of Thorndyke (1977) provides support for the multidimensional theory of narrative comprehension. Thorndyke (1977) demonstrated that readers used generic story structures during the story comprehension process. His work demonstrated that plot is an integral component of the narrative comprehension process. This theory suggests that during 
the production of an unconstrained summary the reader uses story content structure strategy which produces syntactic information that is high in transitivity and encodes into a written statement that represents plot understanding. This research has demonstrated that unconstrained summaries produced more plot representation when compared to constrained summary production.

The works of Schank (1982) provides support for the multidimensional theory of narrative comprehension. Schank (1982) suggests that people categorize world knowledge and narrative understanding through the activation of concrete schemata referred to as memory organizational packets (MOPs). MOPs organize similar episodic stereotypical action schemata rather than abstract schemata in memory. Thus during the production of an unconstrained summary the reader uses a MOPs semantic strategy that produces low transitivity features and encodes into a written statement that contains plot information.

Schank (1982) also suggested that although some of our world knowledge is derived from circumstances of an episode, others lie in a more abstract representation between episodes in an episode referred to as thematic organizational packets (TOPs). TOPs are abstract memory structures. Seifert, Dyer, and Black (1986) demonstrated in their work that abstract memory story comprehension schema is referred to as thematic abstract units (TAUs). During the production of a constrained summary the reader uses semantic TOPs and TAUs strategies that produces high transitivity features and encodes into a written statement that contains abstract thematic information. This research demonstrated that constrained summaries produced more thematic abstract units than unconstrained summaries.

The work of Kintsch and van Dijk (1977) also support the multidimensional theory of narrative comprehension. Kintsch and van Dijk (1977) proposed that there are specific macrorules that readers apply during the summary writing process that represent semantic strategies used to produce summaries. Thus, Kintsch and van Dijk's (1977) model represents macrorules as integrating features between narrative structure (semantic) and transitivity 
(syntax). This theory suggests that there is a high correlation between use of macrorules and summary type (for this reason a separate analysis was unnecessary). It is suggested that during the production of a constrained summary the older reader uses the construction macrorule strategy (Marshall, 1984, 1994) to produce syntactic information that is low in transitivity and during the production of an unconstrained summary the older reader uses the integration and generalization macrorule (Brown \& Day, 1983) strategy to produce syntactic information that is high in transitivity.

The works of Brewer and Lichtenstein (1982), structural affect theory, support the multidimensional theory of narrative comprehension. Structural affect suggests that readers attend to concrete, action and emotion producing elements of narratives. These affect producing features of narratives become most memorable to the readers. This research has demonstrated that an unconstrained summary produces concrete action plot features. These plot features are what the reader considers to be most memorable. This implies that an unconstrained summary promotes affect semantic meaning that produces syntactic features that are high in transitivity.

The work of Many (1991) suggests that readers construct text meaning through different stances. The aesthetic stance represents story meaning that is highly concrete while the efferent stance represents meaning that is generic in nature. This implies during the production of an unconstrained summary the reader uses the aesthetic stance which promotes syntactic information that is high in transitivity and is encoded into the written statement as plot representation while during the production of a constrained summary the reader uses an efferent stance which promotes syntactic information that is low in transitivity and is encoded into the written statement as an abstract theme. In sum, a constrained summary or an unconstrained summary promotes the reader's use of different semantic strategies which in turn produce the use of different syntactic strategies. A constrained summary promotes 
meaning that is abstract while an unconstrained summary promotes meaning that is concrete in nature.

\section{Syntactic Features of Story Comprehension}

The major findings of the syntactic phenomena of transitivity feature patterns provide support for the multidimensional theory of narrative comprehension. Givon (1984) and Hopper and Thompson's $(1980,1984)$ notion of transitivity was supported in relation to narrative summary writing production by the results of this research. The results demonstrated that a constrained summary produced lower transitivity values than an unconstrained summary. This indicates that readers have access to multiple syntax features during the production of different summary tasks. Results of this research demonstrated that in the production of a constrained summary subjects used more low in transitivity features in contrast to the production of a unconstrained summary where subjects used more highly transitive features.

An unconstrained summary due to length produced highly transitive features that included an action/event embodied in a verb that is transferred from a concrete subject to a totally affected concrete object. The use of an unconstrained summary permitted the use of specific or concrete coordination of information while simultaneously producing superficial narrative comprehension that was considered to be prototypically transitive or action/event focused. For instance:

Rex knocked over a lamp.

This example from an unconstrained summary is high in transitivity features. A dynamic verb, knocks that transfers action from an agentive subject, Rex to a totally affected direct object, lamp.

In contrast a constrained summary, due to brevity, produced low in transitivity features including stative, copular or mental state verbs that are not easily transferred from an abstract non-agentive subject to an unaffected abstract object or lack of a direct object. The 
use of a constrained summary permitted abstract coordination of information while simultaneously preventing very superficial narrative comprehension that was considered to be low in transitivity. For instance:

No bet is ever a sure bet.

This example demonstrates a low transitivity syntactic feature. A copular verb is does not transfer action from an abstract non agentive subject, bet to any direct object. The results indicated that older readers use different syntactic strategies during the production of different summary tasks.

A general finding derived from this research suggests that older readers have access to multiple semantic strategies and syntactic features that are used in the production of summaries. During the production of a constrained summary specific concrete semantic strategies are used by the reader which produce the use of specific syntactic features which encode into a written statement. During the production of an unconstrained summary specific semantic strategies are used by the reader which produce the use of specific syntactic features which encode into a written statement. Another important general finding of this research suggests that constrained summaries produced thematic abstract units that were generally low in transitivity features while unconstrained summaries produced plot features that were generally high in transitivity features. Further, it is suggested thematic abstract units and low in transitivity features represent a reader's use of abstract writing strategies. Conversely, it is suggested that plot features and high in transitivity features represent a reader's use of concrete writing strategies.

\section{Implications}

This theory has specific research and practical purposes. First, this research provides a two-fold definition of a summary: (a) A constrained summary is a writing comprehension task that produces abstract narrative understanding and (b) An unconstrained summary is a writing comprehension task that produces concrete narrative understanding. Second, this study 
demonstrated that syntax is important in the study of narrative understanding. Older readers during the narrative writing process used specific semantic strategies and syntactic features that are task dependent. Third, older readers use of concrete writing strategies and abstract writing strategies have implications for the development of writing process strategies. Fourth, the purpose of education is to enlighten the learner and this research provides an empirical and theoretical framework to examine task dependent narrative comprehension written products that give learners the opportunity to express multiple narrative meanings. What is empowering for the teacher and the learner is the preparation of comprehension tasks that serve to enlightened the learner by raising her present level of consciousness. Use of a constrained summary provides such a way to raise the learners level of narrative comprehension consciousness. Fifth, the theory and research associated with this study appear to suggest that there is not a clear distinction between narrative comprehension process and product.

As educators our comprehension process and product focus needs to be in the empirical and qualitative observation of comprehension tasks that raise the readers level of consciousness. As educators we need to permit students to express different narrative understandings. Most important our goal is to realize that readers have available to them different semantic strategies and syntactic features that are used to understand narratives. There is a need to provide instruction and educational opportunities that promote reader semantic and syntactic choices.

The results of this research have been discussed from a semantic, syntactic, and written summary perspective. The theory developed is not considered to be complete. Further examination is needed, especially in the area of sentence structure and lexical markings. The comprehension of narratives is a complicated process. This research begins to examine the multidimensional aspects of how readers comprehend narratives. 
The multidimensional theory of narrative comprehension has been supported by the empirical and theoretical findings of this research. This theory begins to postulate how a concepts of narration is stored in the readers memory and is cognitively constructed by the reader. The general findings of this research emphasize the importance of narrative comprehension tasks that give readers opportunities to make different narrative comprehension semantic and syntactic choices.

\section{Limitations}

Now that the research questions raised at the onset of the research have been answered discussion of the present research is at an end. However, limitations of this research needs to be discussed as it has bearing on any further research in this area.

First here are some limitations of this research:

1. The selection of stories needs to be modified for future research. For this research one of the stories used, "Mrs. Packletide's Tiger" included two major episodes in contrast to "Fate" which included one major episode. This accounted for a story order effect during Phase I of the data collection.

2. One of the problems associated with this research was that product and process cannot be separated. for instance the research demonstrated that a constrained summary contained more highly transitive features. The transitive features were examined as a process and a product.

3. Further subject information needed to be obtained such as subjects g.p.a. and age.

4. The metacognitive questionnaire used in this research was designed to begin to examine the process differences between both summaries. However, it fell short in achieving that goal and only began to delineate rough estimates of the types of strategies used by subjects during the production of both summaries. 


\section{Further Research}

This type of research has led to new insights into the processes and products associated with narrative comprehension. Numerous questions and implications for further research have been raised by this study. A listing of such questions would include:

1. Would there be task dependent developmental differences in the production of transitivity features, macrorules, and story features?

2. Would development of a better constructed metacognitive questionnaire provide insight into the process product distinction?

3. Would use of expository texts produce semantic, syntactic and summary differences between use of a constrained summary and an unconstrained summary?

4. Would using the transitivity feature data to formulate a syntactic teaching strategy and using story feature data with the appropriate summary produce learner abstract thinking and/or concrete thinking?

5. Would examination of previous narrative comprehension research product patterns provide insight into the process product distinction and lead to the development of emancipatory literacy programs?

6. Would examination of sentence structure markings and lexical organization provide insight into the process product distinction and lead to the development of writing instructional programs?

7. Would examination of other syntax phenomena lead to further insights into strategies used for academic and creative writing?

\section{Summary}

This research has four major findings. First, there was a significant difference between transitivity features contained in a constrained summary and an unconstrained summary. Constrained summaries contained a greater number of high transitivity features while unconstrained summaries contained a greater number of low transitivity features. Second, 
there were significant differences between usage of thematic abstract units and plot features in a constrained summary and an unconstrained summary. Constrained summaries contained a greater percentage of thematic abstract units while unconstrained summaries contained a greater percentage of plot features. Third, examination of the data indicated that unconstrained summaries contained canonical story features. Fourth, examination of the data indicated that when a story has two climaxes one abstract in nature and the other concrete in nature an unconstrained summary produces a greater number of concrete plot organized summaries while a constrained summary produces a greater number of abstract summaries.

\section{A Concluding Statement}

Research of the type described in this dissertation is needed in the area of reading study. It is hoped that the insights gained by this research will serve to contribute to the understanding of the complicated process of reading comprehension. These new insights are beneficial to those interested in the process of reading and those interested in the teaching of reading to develop pedagogical tools. 
References

Bartlett, F.C. (1932). Remembering: A study in experimental social psychology. Cambridge: Cambridge University Press.

Brewer, W.F., \& Lichtenstein, E. H. (1982). Stories are to entertain: A structural affect theory. Journal of Pragmatics, 6, $473-486$.

Brown, A. \& Day, J. (1983). Macrorules for summarizing text: The development of expertise. Journal of verbal learning and verbal behavior, $22,1-14$.

Graesser, A., Golding, J., \& Long, D. (1991). Narrative representation and comprehension. In R. Barr, M. Kamil, P. Mosenthal, P. Pearson Editors (Eds.), Handbook of Reading Research Volume II. NY : Longman.

Givon, T. (1984). Syntax: A functional Topological Introduction Volume I \& 2. John Benjamins Publishing Company.

Hopper, P. J. \& Thompson, S.A. (1984). The discourse basis for lexical categories in universal grammar. Language, 60, 703-748.

Hopper, P. J. \& Thompson, S.A. (1980). Transitivity in grammar and discourse. Language, 56, 199-251.

Kintsch, W. \& van Dijk, T. (1978). Toward a model of text comprehension and production. Psychological Review, 85, 363-394.

Many, J. (1991). The effects of stance and age level on children's literacy responses. Journal of Reading Behavior, 23, 61-85.

Marshall, N. (April 1984). The level of summarizing skills for narration and exposition. Paper presented at the annual meeting of the A.E.R.A., New Orleans, LA.

Marshall, N. (April 1994). Setting a research agenda for reader/text interactions. Paper presented at the annual meeting of the S.S.S.R., New Orleans, LA.

Polany-Bowditch L. (1976). Why the whats are when: Mutually contextualized realis of narrative. Berkley syntactic Society. 259-177. 
Rosenblatt, L. M. (1986). The aesthetic transaction. Journal of Aesthetic Education, 20, 122-128.

Schank, R. D. \& Abelson, R. (1977). Scripts, plans, goals, and understanding. Hillsdale, NJ : Erlbaum.

Schank, R. D. (1982). Reminding and memory organization : An introduction to MOPS. In W. G. Lehnert, M. H. Ringle Editors (Eds.), Strategies for natural language processing. Hillsdale, NJ : Erlbaum.

Seifert, C, . Dyer, M. , \& Black, J. (1986). Thematic knowledge in story understanding. Text, 6, 393-426.

Stein, N. L. , \& Glenn, C. G. (1979). An analysis of story comprehension in elementary school children. P.O. Freedle Editor (Eds.), New directions in discourse processing (pp. ). Norwood, NJ : Ablex.

Thorndyke, P. (1977). cognitive structures in comprehension and memory of narrative discourse. Cognitive Psychology, 9 77-110. 
Appendix A

Stories

\section{Mrs. Packletide's Tiger by Sake}

It was Mrs. Packletide's pleasure and intention was that she should shoot a tiger, Not that the lust to kill had suddenly descended on her, or that she felt that she would leave India safer and more wholesome than she had found it, with one fraction less of wild beast per million of inhabitants. The compelling motive for her sudden deviation towards the footsteps of Nimrod was the fact that Loona Bimberton had recently been carried eleven miles in an airplane by an Algerian aviator, and talked of nothing else; only a personally procured tiger-skin and heavy hordes of Press photographs could successfully counter that sort of thing. Mrs. Packletide had already arranged in her mind the lunch she should give at her house in Curzon Street, ostensibly in Loon Bimberton's honor, with a tiger-skin rug occupying most of the foreground and all of the conversation. She had also already designed in her mind the tiger-claw brooch that she was going to give Loona Bimberton on her next birthday. In a world that is supposed to be chiefly swayed by hunger and love Mrs. Packletide was an exception; her movements and motives were largely governed by dislike of Loon Bimberton.

Circumstances proved propitious. Mrs. Packletide had offered a thousand rubles for the opportunity of shooting a tiger without over much risk or exertion, and it so happened that a neighboring village could boast of being the favored rendezvous of an animal of respectable antecedents, which had been driven by the increasing infirmities of age to abandon game-killing and confine its appetite to the smaller domestic animals. The prospect of earning the thousand rupees had stimulated the sporting and commercial instinct of the villagers; children were posted night and day on the outskirts of the local jungle to head the tiger back in the unlikely event of his attempting to roam away to fresh hunting-grounds, and the cheaper kinds of goats were left about with elaborate carelessness to keep him satisfied with his present for the Memsahib's shoot. Mothers carrying their babies home through the hunger 
after the day's work in the field hushed their singing lest they might curtail the restful sleep of the venerable herd-robber.

The great night duly arrived, moonlit and cloudless. A platform had been constructed in a comfortable conveniently placed tree, and there on crouched Mrs. Packletide and her paid companion, Miss Mebbin. A goat, gifted with a particularly persistent bleat, such as even a partially deaf tiger might be reasonable expected to hear on a still night, was tethered at the correct distance. with an accurately sighted rifle and thumb-nail pack of patience cards the sportswoman awaiting the coming of the quarry.

"I suppose we are in some danger?' said Miss Mebbin.

She was not actually nervous about the wild beast, but she had a morbid dread of performing an atom more service than she had been paid for.

"Nonsense," said Mrs. Packletide; "it's a very old tiger. it couldn't spring up here even if it wanted to."

Louisa Mebbin adopted a protective elder-sister attitude towards money in general, irrespective of nationality of denomination. Her energetic intervention had saved man a ruble from dissipating itself in tips in some Moscow hotel, and francs and centimes clung to her instinctively under circumstances stances which would have driven them headlong from less sympathetic hands. Her speculations as to the market depreciation of tiger remnant were cut short by the appearance on the scene of the animal itself. As soon as it caught sight of the tethered goat it lay flat on the earth seemingly less from a desire to take advantage of all available cover than for the purpose of snatching a short rest before commencing the grand attack.

"I believer it's ill" said Louisa Mebbin, loudly in Hindustani, for the benefit of the village headman, who was in ambush in a neighboring tree.

"Hush!" said Mrs. Packletide and at that moment the tiger commenced ambling towards his victim. 
"Now, now!" urged Miss Mebbin with some excitement; "if he doesn't touch the goat we needn't pay for it." (The bait was an extra.)

The rifle flashed out with a loud report, and the great tawny beast sprang to one side and them rolled over in the stillness of death. In a moment a crow of excited natives had swarmed on to the scene, and their shouting speedily carried the glad new to the village, where a thumping of tom-toms took up the chorus of triumph. And their triumph and rejoicing found a ready echo in the heart of Mrs. Packletide; already that luncheon-party in Curzon Street seemed immeasurably nearer.

It was Louisa Mebbin who drew attention to the fact that the goat wad in death-throes from a mortal bullet-wound, while no trace of the rifle's deadly work could be found on the tiger, Evidently the wrong animal had been hit, and the best of prey had succumbed to heart-failure, caused by the sudden report of the rifle, accelerated by senile decay. Mrs. Packletide was pardonably annoyed at the discovery; but at any rate, she was the possessor of a dead tiger, and the villagers, anxious for their thousand rupees, gladly connived at the fiction that she had shot the beast. And Miss Mebbin was a paid companion. Therefore did Mrs. Packletide face the cameras with a light heart and her pictured fame reached from the pages of the Texas Weekly Snapshot to the illustrated Monday supplement of the Novoe Vremya. As for Loon Bimberton, she refused to look at the illustrated paper for weeks, and her letter of thanks for the gift of a tiger-claw brooch was a model of repressed emotions. The luncheon party she declined; there are limits beyond which repressed emotions become dangerous.

from Curzon Street the tiger-skin rug traveled down to the Manor House and was duly inspected and admired by the country, and it seemed a fitting and appropriate thing when Mrs. Packletide went to the County costume Ball in the character of Diana. She refused to fall in however to Clovis's tempting suggestion of a primeval dance party, at which every one should wear the skins of beasts they had recently slain. "I should be in rather a Baby Bunting condition," confessed Clovis, "with a miserable rabbit-skin or two to wrap up in, but then" he 
added, with a rather malicious glance at Diana's proportions, "my Figures is quite as good as that Russian dancing boy's."

"How amused everyone would be if they knew what really happened," said Louis Mebbin a few days after the balls.

"What do you mean?' asked Mrs. Packletide, her face changing color as rapidly as though it were going through a book of patterns before post-time.

"Loona Bimberton would," said Miss Mebbin. Mrs. Packletide's face settled on an unbecoming shade of greenish white.

"You surely wouldn't give me away?" she asked.

"I've seen a week-end cottage near Ddorking that I should rather like to buy," said Miss Mebbin with seeming irrelevance. "Six hundred and eighty freehold. Quite a bargain, only I don't happen to have the money."

Louisa Mebbin's pretty week-end cottage, christened by her "Les fauves," and gay in summer time with its garden borders of tiger lilies, is the wonder and admiration of her friends.

"It is a marvel how Louisa manages to do it," is the general verdict.

Mrs. Packletide indulges in no more big-game shooting. "The incidental expenses are so heavy," she confides to inquiring friends. 


\section{Fate written by Sake}

Rex Dillot was nearly twenty-four, almost good-looking and quite penniless. His mother was supposed to make him some sort of an allowance out of what her creditors allowed her, and Rex occasionally strayed into the ranks of those who earn fitful salaries as secretaries or companions to people who are unable to cope unaided with their correspondence or their leisure. for a few months he had been assistant editor and business manger of a paper devoted to fancy mice, but the devotion had been all on one side, and the paper disappeared with a certain abruptness from club reading rooms and other haunts where it had made a gratuitous appearance, Still Rex lived with some air of comfort and well-being as on can live if one is born with a genius for that sort of thing, and a kingly Providence usually arranged that his weekend invitations coincided with the dates on which his one white dinner waistcoat was in a laundry returned condition of dazzling cleanness. He played most games badly, and was shrewd enough to recognize the fact, but he had developed a marvelously accurate judgment in estimating the play and chances of other people, whether in a golf match, billiard handicap, or croquet tournament. By dint of parading his opinion of such and such a player's superiority with a sufficient degree of youthful assertiveness he usually succeeded in provoking a wager at liberal odd, and he looked to his weekend winnings to carry him through the financial embarrassments of his mid-week existence. The trouble was, as he confided to Clovis Sangrail, that he never had enough available or even prospective cash at his command to enable him to fix the wager at a Figure really worth winning.

"Someday," he said, "I shall come across a really safe thing, a bet that simply can't go astray, and than I shall put it up for all I'm worth, or rather for a good deal more than I'm worth if you sold me up to the last button."

"It would be awkward if it didn't happen to come off," said Clovis.

"It would be more than awkward," said Rex; "it would b e a tragedy. All the same, it would be extremely amusing to bring it off. fancy awaking in the morning with about three 
hundred pounds standing to one's credit. I should go and clear out my hostess' pigeon-loft before breakfast out of sheer good-temper."

"Your hostess of the moment mightn't have a pigeon-loft." said Clovis.

"I always choose hostesses that have, " said Rex; "a pigeon-loft is indicative of a careless, extravagant, genial disposition, such as I like to see around me. People who strew corn broadcast for a lot of feathered inanities that just sit about cooing and giving each other the glad eye in a Louis Quatorze manner are pretty certain to do you well."

"Young Strinnit is coming down this afternoon," said Clovis reflectively; "I dare say you won't find it difficult to get him to back himself at billiards. He plays a pretty useful game, but he's not quite as good as he fancies he is."

"I know one member of the party who can walk around him, " said Rex softly, an alert look coming into his eyes; "that cadaverous-looking Major who arrived last night. I've seen him play at St. Moritz. If I could get Strinnit to lay odds on himself against the Major the money would be safe in my pocket. This looks like the good thing I've been watching and praying for.

"Don't be rash," counseled Clovis, "Strinnit may play up to his self-imagined form once in a blue moon."

"I intend to be rash," said Rex quietly, and the look on his face corroborated his words.

"Are you all going to flock to the billiard-room?" asked Teresa Thendleford, after dinner, with an air of some disapproval and a good deal of annoyance. "I can't see what particular amusement you find in watching two men prodding little ivory balls about on a Table."

"Oh well," said her hostess, "it's a way of passing the time, you know.

"A very poor way, to my mind," said Mrs. Thundleford; "now I was going to have shown all of you the photographs I took in Venice last summer." 
"You showed them to us last night," said Mrs. Cuvering hastily. "Those were the ones I took in Florence. These are quite a different lot.

"Oh well, some time tomorrow we can look at them. You can leave them down in the drawing-room, and than everyone can have a look.

"I should prefer to show them when you are all gathered together, as I have quite a lot of explanatory remarks to make, about Venetial art and architecture, on the same lines as my remarks last night on the Florentine galleries. Also, there are some verses of mine that I should like to read your on the rebuilding of the Campanile, But, of course if you all prefer to watch Major Latton and Mr. Strinnit knocking balls about on a Table --."

"They are both supposed to be first-rate players," said the hostess. "I have yet to learn that my verses and my art cause are of second-rate quality," said Mrs. Thundleford with acerbity. "However, as you all seen bent on watching a silly game, there's no more to be said. I shall go upstairs and finish some writing. Laster on, perhaps, I will come and join you."

To one, at least, of onlookers the game was anything but silly. It was absorbing, exciting, exasperating, nerve-stretching, and finally it grew to be tragic. The Major with the St. Moritz reputation was playing a long way below his form, young Strinnit was playing slightly above his, and had all the luck of the game as well. from the very start the balls seemed possessed by a demon of contrariness; they trundled about complacently for one player, they would go nowhere for the other.

"A hundred and seventy, seventy-four," sang out the youth who was marking. In a game of two hundred and fifty up it was an enormous lead to hold Clovis watched the flush of excitement die away from Dillot's face, and a hard white look take its place.

"How much have you go on?' whispered Clovis. The other whispered the sum through dry, shaking lips. It was more than he or any one connected with him could pay; he had done what he had said he would do. He had been rash.

"Two hundred and six, ninety eight." 
Rex heard a clock strike ten somewhere in the hall, then another somewhere else, and another, and another; the house seemed full of striking clocks. Then in the distance the table clock chimed in. In another hour they would all be striking eleven, and he would be listening to them as a disgraced outcast, unable to pay, even in park the wager he had challenged.

"Two hundred and eighteen, a hundred and three." the game was as good as over. Rex was as good as done for. He longed desperately for the ceiling to fall in, for the house to catch fire, for anything o happened that would put an end to that horrible rolling to and fro of red and white ivory that was jostling him nearer and nearer to his doom.

"Two hundred and twenty-eight, a hundred an seven."

Rex opened his cigarette cast; it was empty. That at least gave him a pretext to slip away from the room for the purpose of refilling it; he would spare himself the drawn-out torture of watching that hopeless game played out to the bitter end. He backed away from the circle of absorbed watchers and made his way up a short stairway to a long, silent corridor of bedroom, each with a guest's name written in a little square on the door. In the hush that reigned in this part of the house he could still hear the hateful click-clock of the balls; if he waited for a few minutes longer he would hear the little outbreak of clapping and buzz of congratulation that would hail Strinnit's victory. On the alert tension of his nerves there broke another sound, the aggressive, wrath-inducing breathing of one who sleeps in heavy after dinner slumber. The sound came from a room just at his elbow; the card on the door bore the announcement. "Mrs. Thundleford." The door was just slightly ajar; Rex pushed it open an inch or two more and looked in. the august Teresa had fall asleep over an illustrated guide to Florentine art galleries; at her side, somewhat dangerously near the edge of the Table, was a reading lamp. If fate had been decently kind to him, thought Rex, bitterly, that lamp would have been knocked over by the sleeper and would have given them something to think of besides billiard matches. 
There are occasions when one must take one's fate in one's hands. Rex took the lamp in his.

"Two hundred and thirty-seven, one hundred and fifteen." Strinnit was at the Table, and the balls lay in good position for him, he had a choice of two fairly easy shots, a choice which he was never to decide. A sudden hurricane of shrieks and a rush of stumbling feet sent everyone flocking to the door. The Dillot boy crashed into the room carrying in his arms the vociferous and somewhat disheveled Teresa Thundleford; her clothing was certainly not a mass of flames, as the more executable members of the party afterwards declared, but the edge of her skirt and part of the Table cover in which she had been hastily wrapped were alight in a flickering, half-hearted manner. Rex flung his struggling burden on to the billiard Table, and for one breathless minute the work of beating out the sparks with rugs and cushions and playing on them with soda water siphons engrossed the energies of the entire company.

"It was lucky I was passing when it happened," panted Rex; "someone had better see to the room, I think the carpet is alight."

As a matter of fact the promptitude and energy of the rescuer had prevented any great damage being done, either to the victim or her surroundings. The billiard Table had suffered the most, and had to be laid up for repairs; perhaps it was not the best place to have chosen for the scene of salvage operations; but then, as Clovis remarked, when one is rushing about with a blazing woman in one's arms one can't stop to think out exactly where one is going to put her. 


\section{Appendix B}

\section{The King and his Shirt}

A king once fell ill.

"I will give half my kingdom to the man who can cure me:, he said

All his wise men gathered together to decide how the king could be cured. But no one knew. Only one of the wise men said what he though would cure the king.

"If you can find a happy man, take his shirt, put it on the king-and the king will be cured."

The king sent emissaries to search for a happy man. They traveled far and wide throughout his whole kingdom, but they could no find a happy man. There was no one who was completely satisfied: if a man was rich he was ailing; if he was healthy he was poor; if he was rich and healthy he had a bad wife; or if he had children they were bad-everyone had something to complain of.

Finally, late one night, the king's son was passing by a poor little hut and he heard someone say:

"Now God be praised, I have finished my work, I have eaten my fill, and I can lie down and sleep! What more could I want?"

The king's son rejoiced and gave orders that the man's shirt be taken and carried to the king, and that the man be given as much money as he wanted.

The emissaries went in to take off the man's shirt, but the happy man was so poor he had no shirt. 
Appendix C

Sample of Experimental Packet

Directions: Read this story. After you have completed the story go on to the next page for further directions.

It was Mrs. Packletide's pleasure and intention was that she should shoot a tiger, Not that the lust to kill had suddenly descended on her, or that she felt that she would leave India safer and more wholesome than she had found it, with one fraction less of wild beast per million of inhabitants. The compelling motive for her sudden deviation towards the footsteps of Nimrod was the fact that Loona Bimberton had recently been carried eleven miles in an airplane by an Algerian aviator, and talked of nothing else; only a personally procured tiger-skin and heavy hordes of Press photographs could successfully counter that sort of thing. Mrs. Packletide had already arranged in her mind the lunch she should give at her house in Curzon Street, ostensibly in Loon Bimberton's honor, with a tiger-skin rug occupying most of the foreground and all of the conversation. She had also already designed in her mind the tiger-claw brooch that she was going to give Loona Bimberton on her next birthday. In a world that is supposed to be chiefly swayed by hunger and love Mrs. Packletide was an exception; her movements and motives were largely governed by dislike of Loon Bimberton.

Circumstances proved propitious. Mrs. Packletide had offered a thousand rubles for the opportunity of shooting a tiger without over much risk or exertion, and it so happened that a neighboring village could boast of being the favored rendezvous of an animal of respectable antecedents, which had been driven by the increasing infirmities of age to abandon game-killing and confine its appetite to the smaller domestic animals. The prospect of earning the thousand rupees had stimulated the sporting and commercial instinct of the villagers; children were posted night and day on the outskirts of the local jungle to head the tiger back in the unlikely event of his attempting to roam away to fresh hunting-grounds, and the cheaper kinds of goats were left about with elaborate carelessness to keep him satisfied with his 
present for the Memsahib's shoot. Mothers carrying their babies home through the hunger after the day's work in the field hushed their singing lest they might curtail the restful sleep of the venerable herd-robber.

The great night duly arrived, moonlit and cloudless. A platform had been constructed in a comfortable conveniently placed tree, and there on crouched Mrs. Packletide and her paid companion, Miss Mebbin. A goat, gifted with a particularly persistent bleat, such as even a partially deaf tiger might be reasonable expected to hear on a still night, was tethered at the correct distance. with an accurately sighted rifle and thumb-nail pack of patience cards the sportswoman awaiting the coming of the quarry.

"I suppose we are in some danger?' said Miss Mebbin.

She was not actually nervous about the wild beast, but she had a morbid dread of performing an atom more service than she had been paid for.

"Nonsense," said Mrs. Packletide; "it's a very old tiger. it couldn't spring up here even if it wanted to."

Louisa Mebbin adopted a protective elder-sister attitude towards money in general, irrespective of nationality of denomination. Her energetic intervention had saved man a ruble from dissipating itself in tips in some Moscow hotel, and francs and centimes clung to her instinctively under circumstances stances which would have driven them headlong from less sympathetic hands. Her speculations as to the market depreciation of tiger remnant were cut short by the appearance on the scene of the animal itself. As soon as it caught sight of the tethered goat it lay flat on the earth seemingly less from a desire to take advantage of all available cover than for the purpose of snatching a short rest before commencing the grand attack.

"I believer it's ill" said Louisa Mebbin, loudly in Hindustani, for the benefit of the village headman, who was in ambush in a neighboring tree. 
"Hush!" said Mrs. Packletide and at that moment the tiger commenced ambling towards his victim.

"Now, now!" urged Miss Mebbin with some excitement; "if he doesn't touch the goat we needn't pay for it." (The bait was an extra.)

The rifle flashed out with a loud report, and the great tawny beast sprang to one side and them rolled over in the stillness of death. In a moment a crow of excited natives had swarmed on to the scene, and their shouting speedily carried the glad new to the village, where a thumping of tom-toms took up the chorus of triumph. And their triumph and rejoicing found a ready echo in the heart of Mrs. Packletide; already that luncheon-party in Curzon Street seemed immeasurably nearer.

It was Louisa Mebbin who drew attention to the fact that the goat wad in death-throes from a mortal bullet-wound, while no trace of the rifle's deadly work could be found on the tiger, Evidently the wrong animal had been hit, and the best of prey had succumbed to heart-failure, caused by the sudden report of the rifle, accelerated by senile decay. Mrs. Packletide was pardonably annoyed at the discovery; but at any rate, she was the possessor of a dead tiger, and the villagers, anxious for their thousand rupees, gladly connived at the fiction that she had shot the beast. And Miss Mebbin was a paid companion. Therefore did Mrs. Packletide face the cameras with a light heart and her pictured fame reached from the pages of the Texas Weekly Snapshot to the illustrated Monday supplement of the Novoe Vremya. As for Loon Bimberton, she refused to look at the illustrated paper for weeks, and her letter of thanks for the gift of a tiger-claw brooch was a model of repressed emotions. The luncheon party she declined; there are limits beyond which repressed emotions become dangerous.

from Curzon Street the tiger-skin rug traveled down to the Manor House and was duly inspected and admired by the country, and it seemed a fitting and appropriate thing when Mrs. Packletide went to the County costume Ball in the character of Diana. She refused to fall in however to Clovis's tempting suggestion of a primeval dance party, at which every one 
should wear the skins of beasts they had recently slain. "I should be in rather a Baby Bunting condition," confessed Clovis, "with a miserable rabbit-skin or two to wrap up in, but then" he added, with a rather malicious glance at Diana's proportions, "my Figures is quite as good as that Russian dancing boy's."

"How amused everyone would be if they knew what really happened," said Louis Mebbin a few days after the balls.

"What do you mean?' asked Mrs. Packletide, her face changing color as rapidly as though it were going through a book of patterns before post-time.

"Loona Bimberton would," said Miss Mebbin. Mrs. Packletide's face settled on an unbecoming shade of greenish white.

"You surely wouldn't give me away?" she asked.

"I've seen a week-end cottage near Ddorking that I should rather like to buy," said Miss Mebbin with seeming irrelevance. "Six hundred and eighty freehold. Quite a bargain, only I don't happen to have the money."

Louisa Mebbin's pretty week-end cottage, christened by her "Les fauves," and gay in summer time with its garden borders of tiger lilies, is the wonder and admiration of her friends.

"It is a marvel how Louisa manages to do it," is the general verdict.

Mrs. Packletide indulges in no more big-game shooting. "The incidental expenses are so heavy," she confides to inquiring friends. 
Directions: Write a one sentence summary on the space provided below about the story you have just read. DO NOT LOOK BACK AT THE STORY. A one sentence summary included: (1) one sentence consisting of five to fifteen words and (2) includes what you consider to be important information about the story you have just read.

GO ON TO THE NEXT PAGE FOR FURTHER INSTRUCTIONS. 
Directions: Please answer the following questions before you go on to the next story. Answer each question on the space provided.

1. What is your native language?

If your native language is not English please answer the following questions.

2. At what age did you learn to speak English? Please place a check mark in the appropriate space and write down the specific age in which you began to learn English.

$0-2$

$2-4$

4-6

$6-8$

$8-10$

$12-14$

other

PLEASE GO ON AND READ THE NEXT STORY 
Rex Dillot was nearly twenty-four, almost good-looking and quite penniless. His mother was supposed to make him some sort of an allowance out of what her creditors allowed her, and Rex occasionally strayed into the ranks of those who earn fitful salaries as secretaries or companions to people who are unable to cope unaided with their correspondence or their leisure. for a few months he had been assistant editor and business manger of a paper devoted to fancy mice, but the devotion had been all on one side, and the paper disappeared with a certain abruptness from club reading rooms and other haunts where it had made a gratuitous appearance, Still Rex lived with some air of comfort and well-being as on can live if one is born with a genius for that sort of thing, and a kingly Providence usually arranged that his weekend invitations coincided with the dates on which his one white dinner waistcoat was in a laundry returned condition of dazzling cleanness. He played most games badly, and was shrewd enough to recognize the fact, but he had developed a marvelously accurate judgment in estimating the play and chances of other people, whether in a golf match, billiard handicap, or croquet tournament. By dint of parading his opinion of such and such a player's superiority with a sufficient degree of youthful assertiveness he usually succeeded in provoking a wager at liberal odd, and he looked to his weekend winnings to carry him through the financial embarrassments of his mid-week existence. The trouble was, as he confided to Clovis Sangrail, that he never had enough available or even prospective cash at his command to enable him to fix the wager at a Figure really worth winning.

"Someday," he said, "I shall come across a really safe thing, a bet that simply can't go astray, and than I shall put it up for all I'm worth, or rather for a good deal more than I'm worth if you sold me up to the last button."

"It would be awkward if it didn't happen to come off," said Clovis.

"It would be more than awkward," said Rex; "it would b e a tragedy. All the same, it would be extremely amusing to bring it off. fancy awaking in the morning with about three 
hundred pounds standing to one's credit. I should go and clear out my hostess' pigeon-loft before breakfast out of sheer good-temper."

"Your hostess of the moment mightn't have a pigeon-loft." said Clovis.

"I always choose hostesses that have, " said Rex; "a pigeon-loft is indicative of a careless, extravagant, genial disposition, such as I like to see around me. People who strew corn broadcast for a lot of feathered inanities that just sit about cooing and giving each other the glad eye in a Louis Quatorze manner are pretty certain to do you well."

"Young Strinnit is coming down this afternoon," said Clovis reflectively; "I dare say you won't find it difficult to get him to back himself at billiards. He plays a pretty useful game, but he's not quite as good as he fancies he is."

"I know one member of the party who can walk around him, " said Rex softly, an alert look coming into his eyes; "that cadaverous-looking Major who arrived last night. I've seen him play at St. Moritz. If I could get Strinnit to lay odds on himself against the Major the money would be safe in my pocket. This looks like the good thing I've been watching and praying for.

"Don't be rash," counseled Clovis, "Strinnit may play up to his self-imagined form once in a blue moon."

"I intend to be rash," said Rex quietly, and the look on his face corroborated his words.

"Are you all going to flock to the billiard-room?" asked Teresa Thendleford, after dinner, with an air of some disapproval and a good deal of annoyance. "I can't see what particular amusement you find in watching two men prodding little ivory balls about on a Table."

"Oh well," said her hostess, "it's a way of passing the time, you know.

"A very poor way, to my mind," said Mrs. Thundleford; "now I was going to have shown all of you the photographs I took in Venice last summer." 
"You showed them to us last night," said Mrs. Cuvering hastily. "Those were the ones I took in Florence. These are quite a different lot.

"Oh well, some time tomorrow we can look at them. You can leave them down in the drawing-room, and than everyone can have a look.

"I should prefer to show them when you are all gathered together, as I have quite a lot of explanatory remarks to make, about Venetial art and architecture, on the same lines as my remarks last night on the Florentine galleries. Also, there are some verses of mine that I should like to read your on the rebuilding of the Campanile, But, of course if you all prefer to watch Major Latton and Mr. Strinnit knocking balls about on a Table -..."

"They are both supposed to be first-rate players," said the hostess. "I have yet to learn that my verses and my art cause are of second-rate quality," said Mrs. Thundleford with acerbity. "However, as you all seen bent on watching a silly game, there's no more to be said. I shall go upstairs and finish some writing. Laster on, perhaps, I will come and join you."

To one, at least, of onlookers the game was anything but silly. It was absorbing, exciting, exasperating, nerve-stretching, and finally it grew to be tragic. The Major with the St. Moritz reputation was playing a long way below his form, young Strinnit was playing slightly above his, and had all the luck of the game as well. from the very start the balls seemed possessed by a demon of contrariness; they trundled about complacently for one player, they would go nowhere for the other.

"A hundred and seventy, seventy-four," sang out the youth who was marking. In a game of two hundred and fifty up it was an enormous lead to hold Clovis watched the flush of excitement die away from Dillot's face, and a hard white look take its place.

"How much have you go on?' whispered Clovis. The other whispered the sum through dry, shaking lips. It was more than he or any one connected with him could pay; he had done what he had said he would do. He had been rash.

"Two hundred and six, ninety eight." 
Rex heard a clock strike ten somewhere in the hall, then another somewhere else, and another, and another; the house seemed full of striking clocks. Then in the distance the table clock chimed in. In another hour they would all be striking eleven, and he would be listening to them as a disgraced outcast, unable to pay, even in park the wager he had challenged.

"Two hundred and eighteen, a hundred and three." the game was as good as over. Rex was as good as done for. He longed desperately for the ceiling to fall in, for the house to catch fire, for anything o happened that would put an end to that horrible rolling to and fro of red and white ivory that was jostling him nearer and nearer to his doom.

"two hundred and twenty-eight, a hundred an seven."

Rex opened his cigarette cast; it was empty. That at least gave him a pretext to slip away from the room for the purpose of refilling it; he would spare himself the drawn-out torture of watching that hopeless game played out to the bitter end. He backed away from the circle of absorbed watchers and made his way up a short stairway to a long, silent corridor of bedroom, each with a guest's name written in a little square on the door. In the hush that reigned in this part of the house he could still hear the hateful click-clock of the balls; if he waited for a few minutes longer he would hear the little outbreak of clapping and buzz of congratulation that would hail Strinnit's victory. On the alert tension of his nerves there broke another sound, the aggressive, wrath-inducing breathing of one who sleeps in heavy after dinner slumber. the sound came from a room just at hi elbow; the card on the door bore the announcement. "Mrs. Thundleford." The door was just slightly ajar; Rex pushed it open an inch or two more and looked in. the august Teresa had fall asleep over an illustrated guide to Florentine art galleries; at her side, somewhat dangerously near the edge of the Table, was a reading lamp. If fate had been decently kind to him, thought Rex, bitterly, that lamp would have been knocked over by the sleeper and would have given them something to think of besides billiard matches. 
There are occasions when one must take one's fate in one's hands. Rex took the lamp in his.

"Two hundred and thirty-seven, one hundred and fifteen." Strinnit was at the Table, and the balls lay in good position for him, he had a choice of two fairly easy shots, a choice which he was never to decide. A sudden hurricane of shrieks and a rush of stumbling feet sent everyone flocking to the door. The Dillot boy crashed into the room carrying in his arms the vociferous and somewhat disheveled Teresa Thundleford; her clothing was certainly not a mass of flames, as the more executable members of the party afterwards declared, but the edge of her skirt and part of the Table cover in which she had been hastily wrapped were alight in a flickering, half-hearted manner. Rex flung his struggling burden on to the billiard Table, and for one breathless minute the work of beating out the sparks with rugs and cushions and playing on them with soda water siphons engrossed the energies of the entire company.

"It was lucky I was passing when it happened," panted Rex; "someone had better see to the room, I think the carpet is alight."

As a matter of fact the promptitude and energy of the rescuer had prevented any great damage being done, either to the victim or her surroundings. The billiard Table had suffered the most, and had to be laid up for repairs; perhaps it was not the best place to have chosen for the scene of salvage operations; but then, as Clovis remarked, when one is rushing about with a blazing woman in one's arms one can't stop to think out exactly where one is going to put her. 
Directions: Write a free-retelling summary on the space provided below about the story you have just read. DO NOT LOOK BACK AT THE STORY. A free-retelling includes: (1) multiple sentences and (2) includes what you consider to be important information about the story you have just read. 
Directions: Please answer the following questions. If you need more space please turn this paper over.

1. How did you choose the information that you included in your free retelling?

2. How did you choose the information that you included in your one sentence summary?

3. What do you consider to be the differences between a free retelling and a one sentence summary?

4. Would you change any of the information in your free retelling?

5. Would you change any of the information in your constrained summary? 
GO ON TO THE NEXT PAGE FOR FURTHER INSTRUCTIONS

You have completed this packet turn it over and wait for further instructions. 
Appendix D

Story Scoring Procedure Samples

Please note that all clauses contain a verb. Thereby, use of a verb in a sentence represents a clausal unit.

Constrained Summary Scoring Procedure Samples

Spiritual things are more important than superficial

Story feature Scoring Procedure

This scoring procedure sample of a constrained summary represents a thematic abstract unit story feature. The summary imparts an adage-like topic sentence that represents abstract thematic narrative understanding.

Unconstrained Summary Scoring Procedure

Rex bets on games. He was poor. He bet on a pool game. He lost money. He went to a room and started a lady on fire. The two players stopped the game.

This scoring procedure sample of an unconstrained summary represents plot story organization. The summary imparts plot narrative understanding. 


\section{Appendix E}

Transitivity Scoring Procedure Samples

Nonprototypical Transitivity Features Scoring Procedure

This scoring procedure sample of a constrained summary represents low transitivity features. Transitivity values are placed after each subject, verb, and direct object. The scoring procedures are as follows:

Spiritual things 1 are 1 more important than superficial

\section{Clause 1}

1. "Spiritual things" represents an abstract, nonagentive, nonvolitional subject and has a transitivity value of 1 .

2. "are" represents a copula verb and has a transitivity value of 1 .

3. There is no direct object in this clause.

First, this clause is a low clause because there is a detransitized verb "are" that is unable to carry an action from the subject to the object. Second, this clause contains a nonagentive abstract subject that cannot affect a direct object even if a direct object were present in the clause. Third, since the verb was a copula verb, "are" there is not direct object in the clause and therefore there is o affectedness and o non-individuation.

Unconstrained Summary Scoring Procedure

This scoring procedure sample of an unconstrained summary represents high transitivity features. Use of a slash represents the end of a clausal unit and the transitivity value is placed after each subject, verb and direct object. The scoring procedures follows:

Rex 3 bets 3 on games 1 . / He 3 was 1 poor. He 3 bet 3 on a pool game 1 . He 3 lost $\mathbf{3}$ money $\mathbf{1}$ and $\backslash$ he $\mathbf{3}$ went $\mathbf{3}$ to a room 1 . He $\mathbf{3}$ started $\mathbf{3}$ a lady on fire $\mathbf{3} \backslash$ The two players $\mathbf{3} \backslash$ stopped 3 the game 3 .

1. "Rex" represents a concrete subject," "bet" represent a dynamic verb and 
"games "represents an unaffected direct object.

2. "He" represents a concrete subject and "was" represents a copular.

3. "He" represents a concrete subject, "bet" represents a dynamic verb and "pool game" represents an unaffected direct object.

4. "He" represents a concrete subject, "lost" represents a dynamic verb and money represents an unaffected direct object.

5. "He" represents a concrete subject, "went" represents a dynamic verb, and "room "represents an unaffected direct object.

6. "He" represents a concrete subject, "started" represents a dynamic verb "lady" was scored as an affected direct object.

7. "The two player" represents concrete subjects, "stopped" represents a dynamic verb and "the game" represents an affected direct object. 
Appendix F Table 1

Constrained Summary Thematic Abstract Unit by Treatment (1-4)

$\begin{array}{lllll}\text { Treatment } & & & & \\ 1 & 2 & 3 & 4 & \\ \text { PC/FU } & \text { PU/FC } & \text { FU/PC } & \text { FC/PU } & \text { Row Total }\end{array}$

STAU

$\begin{array}{lllllll}\text { Not Used } & \# & 6 & 8 & 5 & 6 & 25 \\ & \% & 31.6 & 42.1 & 31.3 & 33.3 & 34.7\end{array}$

\begin{tabular}{lllllll}
\hline Used & $\#$ & 13 & 11 & 11 & 12 & 47 \\
& $\%$ & 68.4 & 57.9 & 68.8 & 66.7 & 65.3 \\
& & & & & & \\
\hline Total & $\#$ & 19 & 19 & 16 & 18 & 72 \\
& $\%$ & 26.4 & 26.4 & 22.2 & 25.0 & 100 \\
\hline
\end{tabular}

$\begin{array}{llll}\text { Chi-Square Test } & \text { Value } & \text { df } & \text { Significance } \\ \text { Pearson } & .64018 & 3 & .88718\end{array}$

Minimum Expected Frequency 5.556

Note. The $U$ represents unconstrained summary, the $C$ represents constrained summary, $P$ represents Mrs. Packletide's Tiger and F represents Fate. 
Appendix F Table 2

Constrained Summary Plot by Treatment (1-4)

Treatment

$\begin{array}{lllll}1 & 2 & 3 & 4\end{array}$

PC/FU PU/FC FU/PC FC/PU Row Total

SP

Not Used \#

\# 13

11

11

13

48

$\% \quad 68.4$

57.9

68.8

72.2

66.7

Used

\# 6

8

5

5

24

$\% \quad 31.6$

42.1

31.3

27.8

33.3

Total

$\begin{array}{ll}\# & 19 \\ \% & 26.4\end{array}$

Value

df

Significance

Pearson

.96546

3

.80961

Minimum Expected frequency 5.333

Note. The $U$ represents unconstrained summary, the $C$ represents constrained summary, $P$ represents Mrs. Packletide's Tiger and F represents Fate. 
Appendix F Table 3

Constrained Summary Theme by Treatment (1-4)

Treatment

$\begin{array}{llll}1 & 2 & 3 & 4\end{array}$

$\begin{array}{lllll}\mathrm{PC} / \mathrm{FU} & \mathrm{PU} / \mathrm{FC} & \mathrm{FU} / \mathrm{PC} & \mathrm{FC} / \mathrm{PU} & \text { Row Total }\end{array}$

ST

$\begin{array}{ccccccc}\text { Not Used } & \# & 18 & 19 & 16 & 17 & 70 \\ & \% & 94.7 & 100.0 & 100.0 & 94.4 & 97.2\end{array}$

\begin{tabular}{lcccccc}
\hline Used & $\#$ & 1 & 0 & 0 & 1 & 2 \\
& $\%$ & 5.3 & 0.0 & 0.0 & 5.6 & 2.8 \\
& & & & & & \\
\hline Total & $\#$ & 19 & 19 & 16 & 18 & 72 \\
& $\%$ & 26.4 & 26.4 & 22.2 & 25.0 & 100
\end{tabular}

Note. Due to the small sample size of used theme $(n=2)$ the chi-square test was not valid. The $U$ represents unconstrained summary, the $C$ represents constrained summary, $P$ represents Mrs. Packletide's Tiger and F represents Fate. 
Appendix F Table 4

Constrained Summary Other by Treatment (1-4)

$\begin{array}{lllll}\text { Treatment } & & & & \\ 1 & 2 & 3 & 4 & \\ \text { PC/FU } & \text { PU/FC } & \text { FU/PC } & \text { FC/PU } & \text { Row Total }\end{array}$

SO

$\begin{array}{lllllll}\text { Not Used } & \# & 19 & 18 & 15 & 18 & 70 \\ & \% & 100.0 & 94.7 & 93.8 & 100.0 & 97.2\end{array}$

Used

\# 0

$\% \quad 0.0$

5.3

6.3

0

2

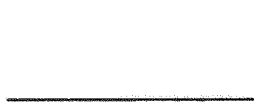

Total

$\begin{array}{ll}\# & 19 \\ \% & 26.4\end{array}$

19

16

18

72

26.4

22.2

25.0

100

Note. Due to the small sample size of used other $(n=2)$ the chi-square text was not valid.

The $U$ represents unconstrained summary, the $\mathrm{C}$ represents constrained summary, $\mathrm{P}$ represents Mrs. Packletide's Tiger and F represents Fate. 
Appendix F Table 5

Unconstrained Summary Thematic Abstract Unit by Treatment (1-4)

$\begin{array}{lllll}\text { Treatment } & & & & \\ 1 & 2 & 3 & 4 & \\ \text { PC/FU } & \text { PU/FC } & \text { FU/PC } & \text { FC/PU } & \text { Row Total }\end{array}$

LTAU

Not Used

\# 17

16

12

14

59

$\% \quad 89.5$

84.2

75.0

77.8

81.9

Used

\# 2

3

4

4

13

$\% \quad 10.5$

15.8

25.0

22.2

18.1

Total

$\begin{array}{ll}\# & 19 \\ \% & 26.4\end{array}$

19

16

18

72

26.4

22.2

25.0

100

Note. Due to small sample size of used thematic abstract unit $(n=13)$ the chi-square text was not valid. The $U$ represents unconstrained summary, the $C$ represents constrained summary, $P$ represents Mrs. Packletide's Tiger and F represents Fate. 
Appendix F Table 6

Unconstrained Summary Plot by Treatment (1-4)

\section{Treatment}

1

2

3

4

$\mathrm{PC} / \mathrm{FU}$

PU/FC

FU/PC

FC/PU

Row Total

LP

Not Used \#

$\% \quad 0.0$

0.0

0.0

5.6

1.4

Used

\# 19

19

16

17

71

$\% \quad 100.0$

100.0

100.0

94.4

98.6

Total

$\begin{array}{ll}\text { \# } & 19 \\ \% & 26.4\end{array}$

19

16

18

72

26.4

22.2

25.0

100

Note. Due to small sample size of unused plot $(n=1)$ the chi-square test was not valid. The

$\mathrm{U}$ represents unconstrained summary, the $\mathrm{C}$ represents constrained summary, $\mathrm{P}$ represents Mrs. Packletide's Tiger and $\mathrm{F}$ represents Fate. 
Appendix F Table 7

Unconstrained Summary Theme by Treatment (1-4)

Treatment

$\begin{array}{lllll}1 & 2 & 3 & 4\end{array}$

PC/FU PU/FC FU/PC FC/PU Row Total

LT

$\begin{array}{lllllll}\text { Not Used } & \# & 19 & 19 & 15 & 14 & 67 \\ & \% & 100.0 & 100.0 & 93.8 & 77.8 & 93.1\end{array}$

\begin{tabular}{lcccccc}
\hline Used & $\#$ & 0 & 0 & 1 & 4 & 5 \\
& $\%$ & 0.0 & 0.0 & 6.3 & 22.2 & 6.9
\end{tabular}

$\begin{array}{lllllll}\text { Total } & \# & 19 & 19 & 16 & 18 & 72 \\ & \% & 26.4 & 26.4 & 22.2 & 25.0 & 100\end{array}$

Note. Due to the small sample size of used theme $(n=5)$ the chi-square text was not valid.

The $U$ represents unconstrained summary, the $C$ represents constrained summary, $P$ represents Mrs. Packletide's Tiger and F represents Eate. 
Appendix F Table 8

Fisher's Exact Test

Unconstrained Theme By Story Order

Unconstrained Theme

PF

FP

Row Total

Not Used

\#

38

28

67

$\%$

100.0

85.3

93.1

Used

\#

0

5

5

$\%$

0.0

14.7

6.9

\section{Total}

\#

38

34

72

$\%$

52.8

47.2

100.0

$\mathrm{p}<.02$, two-tailed.

Note. F represents Fate and P represents Mrs. Packletide's Tiger. 
Appendix F Table 9

Unconstrained Summary Other by Treatment (1-4)

Treatments

$\begin{array}{llll}1 & 2 & 3 & 4\end{array}$

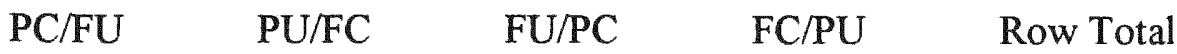

LO

\begin{tabular}{lllllll} 
Not Used & $\#$ & 18 & 18 & 15 & 17 & 68 \\
& $\%$ & 94.7 & 94.7 & 93.8 & 94.4 & 94.4 \\
& & & & & & \\
\hline Used & $\#$ & 1 & 1 & 1 & 1 & 4 \\
& $\%$ & 5.3 & 5.3 & 6.3 & 5.6 & 5.6 \\
\hline Total & $\#$ & 19 & 19 & 16 & 18 & 72 \\
& $\%$ & 26.4 & 26.4 & 22.2 & 25.0 & 100 \\
& & & & & \\
\hline
\end{tabular}

Note. Due to the small sample size of used other $(n=4)$ the chi-square test was not valid.

The $U$ represents unconstrained summary, the $\mathrm{C}$ represents constrained summary, $\mathrm{P}$ represents Mrs. Packletide's Tiger and F represents Fate. 
Appendix F Table 10

Analysis of Variance for Constrained Summary Transitivity by Treatment (1-4)

\begin{tabular}{lcccl} 
Source & df & Sum of Squares & Mean Squares & E \\
\hline Between Groups & 3 & 1.2104 & .4035 & .1851 \\
Within Groups & 68 & 148.1928 & 2.1793 & \\
Total & 71 & & & \\
\hline $\mathrm{p}<.9062$ & & & & \\
\end{tabular}


Appendix F Table 11

Analysis of Variance for Unconstrained Summary Transitivity by Treatment (1-4)

Source

df Sum of Squares Mean Squares F

\begin{tabular}{lrrrr}
\hline Between Groups & 3 & .5670 & .1890 & .6034 \\
Within Groups & 68 & 21.2975 & .3132 & \\
Total & 71 & 21.8644 & & \\
& & & \\
\hline
\end{tabular}

$\mathrm{p}<.6150$ 
Appendix G

Transitivity Variability in Constrained Summaries

There was a greater variability within the constrained summary transitivity feature values as compared to the unconstrained summary transitivity feature values. Examination of the variance suggests specific syntactic/semantic differences between the transitivity features contained within the constrained summaries.

The constrained summary feature value ranges were from 1.5 to 8.0 while the unconstrained summary feature value ranges were from 3.5 to 6.5 . Fourteen of the 33 constrained summaries that contained a transitivity value above the mean value of 4.2 represented thematic abstract units and from a syntactic perspective were considered to be highly transitive. However, a semantic examination of these 14 highly transitive thematic abstract units uncovered an interesting consistent syntactic/semantic pattern. Each of these samples of constrained summary data contained a verb that typically has an agentive subject, but lacked an agentive subject in this case. Some of these constrained summaries which by definition lack agentiveness or volition. In most cases the direct objects were unaffected by the verb. Further, examples $3,4,8,9$, and 13 all contain modal forms. Hopper and Thompsons discussion of modality and transitivity provides the explanation for how modality markers detransitize a clause. Following are some examples:

1. Effortless success does not give you happiness and satisfaction.

In this example "give" syntactically represents a dynamic verb however, the action of "give" cannot be transferred from an abstract subject, "effortless success" to an unaffected generic direct object, "you". This also is a negative which according to Hopper and Thompson is low in transitivity.

2. Greed gets you nowhere but terrible side effects.

3. One person could determine his or her fate in an instance.

4. Power and ambition can kill innocents without any sense. 
5. People change their image according to circumstances.

6. What goes around comes around.

7. You win some you lose some.

8. Some people will do anything to look better than others.

9. Sometimes you have to take fate into your own hands.

10 When fate takes over.

This is a sentence fragment as produced by the subject.

11. Killing a tiger and $\backslash$ the social recognition that came with it.

The second clause is the clause under discussion here.

12. How a person plays with odds.

This is a sentence fragment as produced by the subject.

13. One person could determine his or her fate in an instance.

In each of these examples syntactically a dynamic verb cannot be transferred from an abstract subject to an unaffected generic direct object. Semantically, the dynamic verb does not denote a dynamic action.

Each of these constrained summaries syntactically contained a dynamic verb, an abstract nonagentive subject, and an unaffected abstract direct object. The dynamic verbs in each of these constrained summaries semantically does not represent a concrete action but, rather an abstract representation of an abstract action. 
1950

1979

1982

$1971-1973$

$1974-1979$

$1979-1984$

$1984-1990$

$1990-1996$

1996 - present
Born, Englewood, New Jersey

B.A., Learning Disabilities Florida Atlantic University Boca Raton, Florida

M.S., Reading

Nova University

Davie, Florida

Preschool Teacher

Westside Montessori Teacher

New York, New York

Preschool Teacher

L'Academie Montessori

Ft. Lauderdale, Florida

Learning Disabilities Teacher Reading Teacher

Dillard High School

Ft. Lauderdale, Florida

Reading Teacher

Reading Resource Specialist

Stranahan High School

Ft. Lauderdale, Florida

Graduate Assistant

Preservice Reading Courses

Florida International

University

Miami, Florida

Visiting Instructor

Preservice Reading Courses

Florida International

University

Miami, Florida 